INSTITUTO DE PESQUISAS ENERGÉTICAS E NUCLEARES

Autarquia associada à Universidade de São Paulo

\title{
UM ESTUDO SOBRE MÉTODOS DE CÁLCULO E MEDIDAS \\ EXPERIMENTAIS DE PARÂMETROS CINÉTICOS EM SISTEMAS \\ SUBCRÍTICOS ACIONADOS POR FONTE
}

SEUNG MIN LEE

Dissertação apresentada como parte dos requisitos para obtenção do grau do Mestre na Área de Concentração em Reatores Nucleares de Potência

Orientador: Prof. Dr. José Rubens Maiorino 
INSTITUTO DE PESQUISAS ENERGÉTICAS E NUCLEARES

Autarquia associada à Universidade de São Paulo

\section{A STUDY OF CALCULATION METHODOLOGY AND \\ EXPERIMENTAL MEASUREMENTS OF THE KINETIC \\ PARAMETERS FOR SOURCE DRIVEN SYSTEMS}

SEUNG MIN LEE

Dissertação apresentada como parte dos requisitos para obtenção do grau do Mestre na Área de Concentração em Reatores Nucleares de Potência

Orientador: Prof. Dr. José Rubens Maiorino 
A Sung Sil, minha mãe, pela paciência e pelo apoio incondicional. 


\section{AGRADECIMENTOS}

Ao Prof. Dr. José Rubens Maiorino pela orientação, paciência e dedicação.

Ao Prof. Dr. Admir dos Santos, do Centro de Engenharia Nuclear (CEN), pelas importantes observações e informações.

Ao Prof. Dr. Piero Ravetto, pelo curso sobre Dinâmica de Reatores, que despertou meu interesse pela linha de pesquisa.

A Pedro C. R. Rossi e os demais colegas da pós-graduação do CEN pelo apoio inesquecível e companheirismo.

A Ricardo Diniz e Renato Kuramoto pelas valiosas informações sobre os métodos experimentais de medidas.

Ao Dr. Lamartine Nogueira Frutuoso Guimarães e ao Dr. Alexandre Caldeira do Instituto de Estudos Avançados (IEAv), pelas importantes observações.

À toda a equipe do CEN pelo apoio.

Ao Instituto de Pesquisas Energéticas e Nucleares (IPEN) e a secretaria da Pósgraduação pelo apoio institucional.

À Agência Internacional de Energia Atômica (AIEA) pelo suporte parcial através do Contrato de Pesquisa RC 13388. 


\section{RESUMO}

Este trabalho apresenta um estudo teórico sobre a cinética de reatores focando à metodologia de cálculo e às medidas experimentais dos chamados parâmetros cinéticos para sistemas subcríticos acionados por fontes. São apresentadas, por motivo de comparação, duas metodologias de cálculo dos parâmetros cinéticos: a metodologia baseada no formalismo de Dulla, e a clássica. O objetivo é mostrar a dependência desses parâmetros com o grau de subcriticalidade e a perturbação. Foram considerados um sistema rápido e um térmico, ambos de tipo placa e de meio homogêneo. O modelo utilizado foi de difusão e as soluções foram obtidas usando o método de expansão, considerando 1 grupo de energia e 1 família de precursores para o sistema rápido, e 2 grupos de energia e 1 família de precursores para o sistema térmico. São apresentadas, também, descrições dos principais métodos de medidas experimentais dos parâmetros cinéticos, i.e., métodos estáticos e de fonte pulsada, visando levantar a discussão sobre a compatibilidade desses método na região de subcriticalidade. 


\begin{abstract}
This work presents a theoretical study of reactor kinetics focusing on the methodology of calculation and the experimental measurements of the so-called kinetic parameters. A comparison between the methodology based on the Dulla's formalism and the classical method is made. The objective is to exhibit the dependence of the parameters on subcriticality level and perturbation. Two different slab type systems were considered: thermal one and fast one, both with homogeneous media. One group diffusion model was used for the fast reactor, and for the thermal system, two groups diffusion model, considering, in both case, only one precursor's family. The solutions were obtained using the expansion method. Also, descriptions of the main experimental methods of measurements of the kinetic parameters are presented in order to put a question about the compatibility of these methods in subcritical region.
\end{abstract}




\section{SUMÁRIO}

\section{INTRODUÇÃO}

1.1. O Estado da Arte dos Sistemas Subcríticos Acionados por Fontes (ADS)

1.2. Revisão da Literatura sobre os Parâmetros Cinéticos em Sistemas Subcríticos Acionados por Fontes

1.3. Objetivo e Divisão do Trabalho

\section{METODOLOGIA DE CÁLCULO DE PARÂMETROS CINÉTICOS}

2.1. A Equação de Transporte e o Modelo de Difusão

2.2. A Cinética Pontual e os Parâmetros Cinéticos

2.3. Problemas resolvidos

2.3.1. Modelo de 1 grupo de energia

2.3.2. Modelo de 2 grupos de energias

\section{MÉTODOS EXPERIMENTAIS DE MEDIDAS DE PARÂMETROS CINÉTICOS}

3.1. Método da Fonte de ${ }^{252} \mathrm{Cf}$

3.2. Método das Desidades Espectrais

3.3. Método Rossi- $\alpha$

3.4. Método de Número de Nelson

3.5. Método Feynman- $\alpha$

3.6. Método de Bennet ou da Covariância

3.7. Métodos de Fonte de Nêutrons Pulsada

3.7.1. Método de "Slope Fit" ou Método de Ajuste de Inclinação

3.7.2. Método de Sjöstrand ou Método das Áreas

3.8. Experiência de "Source Jerk"

3.9. Método da Reatividade Inversa

4. CONCLUSÃO E RECOMENDAÇÕES

\section{REFERÊNCIAS BIBLIOGRÁFICAS}




\section{APÊNDICE}

1. TABELAS DOS RESULTADOS

2. OPERADORES ADJUNTOS

3. TEORIA DA PERTURBAÇÃO 


\section{INTRODUÇÃO}

\subsection{O estado da arte dos Sistemas Subcríticos Acionados por Fontes (ADS)}

Os sistemas subcríticos acionados por uma fonte de nêutrons externa de um acelerador, também denominados ADS (Accelerator Driven System), tem sido internacionalmente reconhecidos como potencialmente o reator inovador dedicado à transmutação de resíduos de alta atividade (HLW), para consequentemente reduzir os requisitos nos repositórios geológicos[1 - 4]. O Estado da Arte de ADS tem sido amplamente reportado $[5,6]$

Um ADS consiste de um sistema rápido subcrítico mantido em estado estacionário por uma fonte externa de nêutrons. Na Figura 1, ilustra-se o conceito básico de um ADS, o qual em resumo consiste de:

1) Acelerador (Cíclotron ou Linac), o qual aceleram o feixe de partículas (e.g. prótons);

2) o alvo, onde os nêutrons são produzidos por "spallation";

3) refrigerante(loop ou piscina), sódio, chumbo, ou chumbo-bismuto fundidos, ou gás(Hélio); e

4) o núcleo subcrítico ( $\mathrm{k}_{\text {eff }} \sim 0,95-0,97$ ), consistindo de combustíveis em forma metálica, óxido, ou nitreto, com material nuclear (U, Th, Pu, Actinídeos Menores, $\mathrm{Am}, \mathrm{Np}, \mathrm{Cu}$, etc.), onde energia é gerada com um ganho positivo, e incineração dos actinídeos menores é possível, além de regeneração (U/Pu; Th/U). 


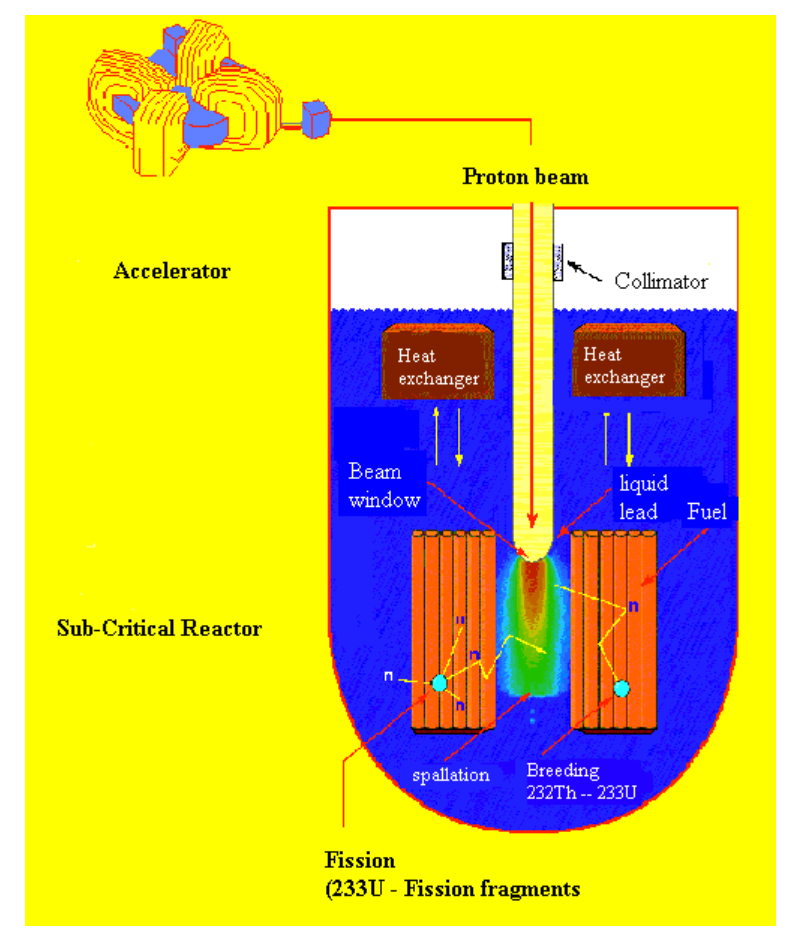

Figura 1. Conceito Básico do ADS

Uma revisão do estado da arte descrevendo os vários projetos em andamento foi apresentada na dissertação do mestrado de Antunes [7].

Atualmente não existe nenhum ADS de potência em operação, apesar de vários projetos terem sido propostos, destacando-se o projeto Belga MYRRHA [8]. Por outro lado várias instalações experimentais de potência zero foram construídas, ou estão em planejamento com o objetivo principal da medida de parâmetros de Física de Reatores, e em especial os parâmetros cinéticos. Dentre as várias instalações experimentais, destaca-se o experimento MUSE, realizado no Reator de Potência Zero MASURCA [9,10].

Outras instalações experimentais estão praticamente operando, com destaque as instalações YALINA-BOOSTER, na Belarus [11], e o experimento ADSR (Accelerator Driven Subcritical Reactor), no Reator de Potência Zero da Universidade de Kyoto (KURRI), Japão[12]. Todas estas instalações, atualmente fazem parte do Projeto Coordenado de Pesquisa da Agência Internacional de Energia Atômica (AIEA), "IAEA Coordinated Research Project (CRP) on Analytical and Experimental Benchmark Analysis of ADS" [13], que tem como objetivo a validação de métodos de cálculo de sistemas subcríticos acionados 
por fonte, através da comparação dos resultados experimentais obtidos nestas instalações com os resultados obtidos por simulação. Em particular o IPEN participa deste CRP com um contrato de pesquisa com a AIEA. Mais além, no Reator Brasileiro de Potência Zero, IPENMB-01, desde 1988, tem-se realizado medidas de parâmetros de Física de Reatores. Recentemente uma proposta foi apresentada para a introdução de um gerador de nêutrons compacto numa configuração subcrítica do IPEN-MB-01 [14], e uma nova técnica de medida de parâmetros cinéticos em configurações subcríticas do IPEN-MB-01 foi desenvolvida, como parte de tese de doutoramento no IPEN [15].

Por outro lado, as técnicas de medidas de parâmetros cinéticos, em muitos casos são baseadas em modelos simplificados (cinética pontual), e baseadas no estado crítico do reator. Mais além, a maioria dos métodos de cálculos destes parâmetros é baseada em modelos desenvolvidos para sistemas críticos. Entretanto conforme apontado por Dulla et al. [16], a definição de parâmetros cinéticos de sistemas subcríticos acionados por fonte, ainda necessita uma investigação mais aprofundada, na medida em que conforme demonstrado teoricamente por esta, estes parâmetros variam com o grau de subcriticalidade, e com a perturbação introduzida para cálculo destes parâmetros.

Como apontado por Dulla et. al. [17], a definição dos parâmetros integrais para sistemas subcríticos acionados por fontes externas, precisa ser repensada. A seguir é apresentado um resumo da primeira parte do trabalho.

Parafraseando o documento apresentado por Dulla et al. [17]:

"Parâmetros integrais são usados para dar informações abrangentes sobre as características físicas de um sistema multiplicativo. Porém, alguns parâmetros integrais podem levar a interpretações diferentes dependendo dos objetivos para os quais são introduzidos, que é o caso dos parâmetros cinéticos, que, enquanto são bem determinados para sistemas inicialmente críticos, são abertos à ambigüidade para com os sistemas subcríticos acionados por fontes. 
Portanto, é importante perceber que a metodologia de tratamento dos sistemas subcríticos deve ser inteiramente repensada, e que qualquer generalização direta da aproximação teórica, elaborada para sistemas incialmente críticos, (como na metodologia clássica de Henry) deve ser evitada.

Vários trabalhos foram consagrados para fazer aproximações a partir de diferentes pontos de vista do problema. O trabalho de Favorite e Stacey [18,19] tratou o problema considerando especialmente o aspecto computacional referindo a um esquema variacional matematicamente consistente. A avaliação de diferentes definições de reatividade foi analizada na Referência [20]. As diferentes maneiras de derivar modelos cinéticos foram analizados na 16. Dois trabalhos de Gandini [21,22] lidam com o problema em uma base auto-consistente, analizando os problemas da cinética dos sistemas subcríticos de uma maneira abrangente, através de uma aproximação heurística e generalizada da teoria da perturbação. Nesses dois trabalhos e no outro[23], são dadas as definições de parâmetros cinéticos, introduzindo, em particular, um termo generalizado da reatividade baseado na teoria da perturbação.

A utilidade e a necessidade da cinética pontual deveriam ser repensadas, desde que, ela é muito simples e insuficiente para uma análise realística de um sistema subcrítico, que deveria ser analizada através da solucão direta das equações dependentes do tempo, energia e espaço. No aspecto computacional, o mérito da cinética pontual está diminuindo cada vez mais, graças ao avanço tecnológico. Porém, em outras áreas de engenharia, como por exemplo, térmica e hidráulica, parâmetros integrais continuam sendo úteis, pois eles fornecem informações imediatas e abrangentes sobre comportamento dos sistemas.

$O$ fator de multiplicação $K_{\text {eff, }}$ que é o principal parâmetro integral, quantifica efetivamente a distância do estado do sistema da criticalidade, e está ligado com a reatividade, ou, classicamente, o "desvio da criticalidade”, $\left(K_{\text {eff }}-1\right) / K_{\text {eff. }}$ Este parâmetro contém significado matemático como autovalor da equação do balanço do estado crítico e depende somente das características físicas do sistema. Entretanto, o parâmetro não contém nenhuma informação sobre a fonte do sistema subcrítico em estado estacionário cuja distribuição neutrônica é completamente diferente da de um sistema crítico. Mais além, os resultados gerados em sistemas subcríticos acionados por fontes são, obviamente, 
dependentes da fonte, mas, como já foi mencionado, $K_{\text {eff }}$ não depende da fonte. Portanto, é requerida uma formulação mais adequada para caracterizar a multiplicação intrínseca do sistema".

Dulla introduziu um modelo de cinética para sistemas subcríticos que tem a mesma estrutura do modelo de cinética pontual clássica e que é consistente com o conceito de $\mathrm{K}_{\mathrm{s}}$ [16]. Este modelo será apresentado na Seção 2.2.

Dentro deste contexto enquadra-se o presente trabalho, que objetiva fazer um levantamento do estado da arte das técnicas experimentais de medidas desses parâmetros, e mostrar que o cálculo destes parâmetros em sistemas subcríticos acionados por fonte varia com a subcriticalidade. Este estudo poderá subsidiar uma crítica aos atuais métodos experimentais e propor novos métodos que seriam implementados em outros trabalhos. 


\subsection{Revisão da Literatura sobre os Parâmetros Cinéticos em Sistemas Subcríticos Acionados por Fontes}

O trabalho de Henry [24] é um dos primeiros dedicados à cinética de reatores. O trabalho apresenta a aplicação da teoria da cinética de reatores para se resolver o problema de difusão em física de reatores para experimentos pulsados.

Keepin [25], no seu livro clássico, discute sobre a solução das equações da cinética pontual e sobre alguns aspectos experimentais. As equações de cinética pontual foram derivadas para o estudo da cinética de reatores assumindo o meio infinito, e função forma independente do tempo. No livro encontra-se as primeiras observações sobre a estabilidade de problemas de física de reatores, considerando nêutrons atrasados.

Existem duas maneiras de resolver o problema de difusão de multigrupo: primeiro, o método modal, que consiste em tratar a parte espacial separadamente e depois trabalhar a parte temporal pelas amplitudes modais; e, segundo, o método nodal, que após o tratamento da parte espacial trabalha pelas constantes de acoplamento nodais.

O trabalho de Lewins, publicado em 1960, discute sobre o conceito de função adjunta com dependência temporal [26], levando em conta a importância de nêutrons e precursores, definindo as equações de difusão e as condições de fronteira para a importância neutrônica, particularmente para um sistema fora da criticalidade.

Foderaro e Garabedian [27] obtiveram solução do problema de difusão em multigrupo para sistemas homogêneos críticos. Lewins, em 1961, reduziu as equações de difusão em equações diferenciais ordinária, levando em conta fonte externa [28]. Em 1968, foi proposta por Becker [29] uma formulação geral da cinética pontual, considerando sistemas cujas funções-forma dependem do tempo. 
Em 1974, foi apresentada por Ohtani et al. [30] uma solução para o problema de difusão de um reator tipo placa, independentemente das condições de contorno, usando o método de expansão em séries de Fourier.

Em 1984 [31], foi obtida uma solução analítica para sistemas térmicos utilizando a técnica de transformada de Laplace, considerando os efeitos da migração neutrônica durante o processo de moderação, e usando um "kernel" que leva a uma integral na equação do balanço neutrônico correspondente à razão de nêutrons térmicos dentro do espaço de fase, devido às fissões térmicas.

Em 1986, Lee e Rottler [32] apresentaram soluções analíticas para o problema de difusão em multigrupo. Foram considerados reatores tipo placa e esférico com e sem refletores e com uma fonte externa dependente do espaço. $\mathrm{O}$ sistema de equações diferenciais parciais foi reduzido a um conjunto de equações diferencias, e as equações foram resolvidas gerando uma solução matricial geral para o fluxo neutrônico.

Gandini [21] apresentou em 2001, uma teoria da perturbação generalizada “Heuristically-based generalized perturbation theory (HGPT)", levando em conta o conceito de função importância e o conceito de reatividade generalizada. Neste trabalho foi derivada uma nova cinética pontual e foram definidos novos termos que aparecem a partir da redução da cinética espacial para a pontual.

Dulla et al. [33] aplicaram o método quase-estático para o estudo dos ADS. Este método é eficiente nos problemas com dependência temporal e está baseado na fatorização da densidade neutrônica no produto de uma função forma e uma função amplitude. Dulla et al. apresentaram, em 2005, um trabalho sobre a importância teórica espacial e temporal da fonte externa para modelos simplificados [34], resolvendo as equações de cinética espacial pelo método de expansão e considerando sistemas inicialmente em estado estacionário.

Em 2006, um trabalho sobre a teoria assintótica do espaço sobre um modelo adequado para se estudar experimentos pulsados em sistemas neutrônicos multiplicativos foi apresentado por Dulla et al. [35]. Foi mostrada a utilidade deste modelo para se obter uma solução analítica para problemas de propagação neutrônica, e estas soluções permitiram 
identificar os efeitos nos fenômenos decorrentes da escolha de modelo, mostrando as limitações do modelo de difusão.

Dulla et al. [36] apresentoram uma metodologia para ressolver problemas de difusão em multigrupo em forma fechada para sistemas subcríticos. No trabalho foram apresentadas soluções considerando-se um sistema típico do experimento Yalina Booster.

Em trabalho anterior [7] tratou-se das duas metodologias de cálculo de parâmetros físicos estáticos e cinéticos, e a utilização do código determinístico de transporte ANISN para o cálculo destes parâmetros. Na dissertação de Fernando [37], foi apresentada uma solução analítica obtida pelo método de expansão para cinética espacial, usando o modelo de difusão para sistemas subcríticos acionados por fontes, considerando meios homogêneos. No presente trabalho foca-se no método de cálculo dos parâmetros cinéticos sem precisar resolver equações de cinética.

Medidas de parâmetros cinéticos em sistemas acionados por fontes tem sido realizadas com o objetivo da validação de metodologias de cálculos (parâmetros de validação). Assim, em 1995, uma série de experimentos chamados MUSE foram realizados na instalação MASURCA. O princípio deste experimento era a hipótese da separabilidade dos efeitos da fonte e da multiplicação no núcleo subcrítico. Entre eles os principais foram os experimentos MUSE-3 e MUSE-4. O experimento MUSE-3 consistiu na introdução de uma fonte pulsada de nêutrons, reação nuclear (D-T), no centro do núcleo em diferentes configurações subcríticas. Medidas de parâmetros cinéticos foram realizadas nesse experimento MUSE [38].

Atualmente, medidas dos parâmetros cinéticos estão sendo realizadas dentro do Projeto CRP, como na instalação YALINA-BOOSTER [39], e dentro do CRP, novas medidas estão sendo realizadas e, também, no experimento KUCA. Todas essas medidas utilizam as técnicas clássicas de medir parâmetros cinéticos, que serão discutidas no Capítulo 3. 


\subsection{Objetivos e Divisão do Trabalho}

Neste trabalho, pretende-se, baseado no formalismo teórico de Dulla, calcular os parâmetros cinéticos, para problemas em geometria plana, com um grupo de energia de nêutrons prontos e atrasados, com uma fonte plana em $\mathrm{x}=0$, em um sistema subcrítico, com espalhamento isotrópico, sem considerar a realimentação ou "feedback", e utilizando o modelo de difusão, tendo como objetivo mostrar a dependência desses parâmetros com o grau de subcriticalidade do sistema e a perturbação causada no sistema. A seguir, ainda em geometria plana, o formalismo será estendido para 2 grupos de energia e 1 família de precursores, para o modelo de difusão.

Note que o método analítico para solucionar este problema em estado estacionário, pode facilmente ser obtido através da metodologia apresentada na Referência 37, isto é, método de expansão.

Estes resultados poderão incluir a lista de "Benchmarks" Analíticos que estão sendo definidos no Projeto Coordenado de Pesquisa, CPR, descrito na introdução, de maneira que as várias instituições internacionais possam comparar seus métodos de cálculo destes parâmetros com o proposto por Dulla.

Na primeira seção do Capítulo 2 será apresentada a equação que descreve variações na distribuição da população neutrônica em um reator, isto é, a Equação de Transporte e sua versão mais simples, o modelo de Difusão. A justificativa para a aproximação ao modelo de difusão, sua utilidade e limitações serão discutidas ainda nessa seção. Na Seção 2.2 será discutido um desenvolvimento analítico para o cálculo dos parâmetros cinéticos.

O formalismo será aplicado, inicialmente, usando o modelo de 1 grupo de energia, e depois, usando o de 2 grupos de energia, em problemas ideais cujas constantes físicas estão disponíveis na literatura. Os gráficos das Seções 2.4.1 e 2.4.2 mostram que os parâmetros variam com o grau de subcriticalidade do sistema e com a perturbação aplicada nele. 
No Capítulo 3 serão apresentados descrições de alguns métodos experimentais de medida dos parâmetros cinéticos: métodos estáticos e de fonte pulsada. A Tabela 1 mostra os principais métodos dessas medidas. Além disso, no mesmo capítulo será discutida a compatibilidade de cada um desses métodos com os sistemas subcríticos acionados por fontes.

\begin{tabular}{|c|c|c|c|c|}
\hline Tipo de fonte & \multicolumn{2}{|c|}{ Método de análise } & $\begin{array}{l}\text { Parâmetros } \\
\text { experimentais }\end{array}$ & Parâmetros previamente calculados \\
\hline \multirow{4}{*}{ Fonte intrínseca } & \multicolumn{2}{|c|}{ Método de Referência } & $\rho_{\mathrm{s}}$ & Parâmetros cinéticos $\left(\alpha_{i}, \lambda_{i}\right)+$ fatores MSM \\
\hline & \multicolumn{2}{|c|}{ Método de Rossi- $\alpha$} & \multirow{3}{*}{$\alpha_{p}$} & \\
\hline & \multirow{2}{*}{\multicolumn{2}{|c|}{$\begin{array}{l}\text { Método de Feynman- } \alpha \\
\text { Método de APSD e CPSD }\end{array}$}} & & \\
\hline & & & & \\
\hline \multirow{2}{*}{$\begin{array}{l}\text { Fonte de }{ }^{252} \mathrm{Cs} \\
\text { externa }\end{array}$} & \multicolumn{2}{|c|}{ Método de Rossi- $\alpha$} & $\alpha_{p}$ & \\
\hline & \multicolumn{2}{|c|}{ Método de "Source jerk" } & $\rho_{\mathrm{s}}$ & Parâmetros cinéticos $\left(\alpha_{\mathrm{i}}, \lambda_{\mathrm{i}}\right)$ \\
\hline \multirow{8}{*}{$\begin{array}{l}\text { Fonte pulsada } \\
\text { de nêutrons }\end{array}$} & \multirow{3}{*}{ Técnica de PNS } & M. das Áreas & $\rho_{\mathrm{s}}$ & \\
\hline & & M. Slope fit & $\alpha_{p}$ & \\
\hline & & $\mathrm{M}$ de $\mathrm{K}_{\mathrm{p}}$ & $\mathrm{K}_{\mathrm{p}}$ & Distribuição calculada de $\mathrm{P}(\mathrm{t})$ \\
\hline & \multicolumn{2}{|c|}{ Método de Rossi- $\alpha$} & \multirow{4}{*}{$\alpha_{\mathrm{p}}$} & \\
\hline & \multirow{2}{*}{$\begin{array}{l}\text { Método de } \\
\text { Feynman- } \alpha\end{array}$} & Modo determinístico & & \\
\hline & & Modo estocástico & & \\
\hline & \multicolumn{2}{|c|}{ Método de APSD e CPSD } & & \\
\hline & \multicolumn{2}{|c|}{ Método de variação e frequência } & $\rho_{\mathrm{s}}$ & \\
\hline
\end{tabular}

Tabela 1. Resumo das técnicas de medida de parâmetros cinéticos utilizadas em sistemas subcríticos acionados por fonte.

No último capítulo são apresentadas a conclusão e algumas recomendações. No Apêndice 2 será apresentada uma discusão sobre operadores adjuntos, e no Apêndice 3 uma breve descrição da Teoria da Perturbação. 


\section{METODOLOGIA DE CÁlCULO DE PARÂMETROS CINÉTICOS}

\subsection{A Equação de Transporte e o Modelo de Difusão}

As taxas de reações nucleares em um reator são determinadas pela distribuição de nêutrons dentro do núcleo (do reator). Portanto, o problema central da teoria de reatores é o de determinar essa distribuição. Diz-se que um nêutron é "transportado" quando este sofre qualquer mudança no espaço de fase, definido pela posição, a energia e a direção do movimento do nêutron, e a Teoria de Transporte estuda esse fenômeno. A equação que governa o fenômeno é chamada Equação Linear de Transporte, que é uma forma linearizada da equação de Boltzman da teoria cinética de gases. A equação de transporte na presença de nêutrons atrasados pode ser escrita como [40]:

$$
\begin{array}{r}
\frac{1}{v} \frac{\partial \Phi(\boldsymbol{r}, E, \Omega, t)}{\partial t}=L(t) \Phi(\boldsymbol{r}, E, \Omega, t)+M_{p}(t) \Phi(\boldsymbol{r}, E, \Omega, t) \\
+\sum_{i=1}^{6} \frac{\lambda_{i} \chi_{i}(E)}{4 \pi} C(\boldsymbol{r}, t)+S(\boldsymbol{r}, E, \Omega, t) \\
(i=1,2,3, \ldots),
\end{array}
$$

e

$$
\frac{\partial\left(\frac{\lambda_{i} \chi_{i}(E)}{4 \pi} C(\boldsymbol{r}, t)\right)}{\partial t}=M_{d i}(t) \Phi(\boldsymbol{r}, E, \Omega, t)-\frac{\lambda_{i} \chi_{i}(E)}{4 \pi} C(\boldsymbol{r}, t)
$$

onde as variáveis destas equações têm a definição e significado clássicos utilizados em Física de Reatores.

O termo $C$ é a concentração dos precursores de nêutrons atrasados, $\lambda_{i}$ a constante de decaimento dos precursores da família $i, L$ o operador de remoção ("streaming" + taxa de reação - espalhamento), $M_{p}$ de multiplicação pronta, $M_{d i}$ de multiplicações atrasadas, e $S$ a fonte externa. Esses operadores dependem de modelo; para o modelo de transporte são 
expressos da seguinte forma [40]:

$$
\begin{aligned}
& L(t)=-\Omega \cdot \nabla \ldots-\Sigma_{t}(\boldsymbol{r}, E) \ldots+\int_{0}^{\infty} d E^{\prime} \int_{4 \pi} d \Omega^{\prime} \Sigma_{s}\left(\boldsymbol{r}, E^{\prime} \rightarrow E, \Omega^{\prime} \cdot \Omega, t\right) \ldots, \\
& M_{p}(t)=\sum_{j} \frac{\chi_{p}^{j}(E)}{4 \pi} \int_{0}^{\infty} d E^{\prime} \int_{4 \pi} d \Omega^{\prime}\left(1-\beta^{j}\right) v^{j}\left(E^{\prime}\right) \Sigma_{f}^{j}\left(\boldsymbol{r}, E^{\prime}\right) \ldots,
\end{aligned}
$$

$\mathrm{e}$

$$
M_{d i}(t)=\sum_{j} \frac{\chi_{i}^{j}(E)}{4 \pi} \int_{0}^{\infty} d E^{\prime} \int_{4 \pi} d \Omega^{\prime} \beta_{i}^{j} v^{j}\left(E^{\prime}\right) \Sigma_{f}^{j}\left(r, E^{\prime}\right) \ldots \cdot
$$

A equação de transporte é fácil de se derivar e de se entender, podendo ser interpretada como um balanço da população neutrônica num elemento de volume no espaço de fases, e possui solução para dadas condições de contorno e condição inicial. A solução é o fluxo angular $\Phi(\boldsymbol{r}, E, \Omega, t)$ que contém praticamente toda informação sobre o comportamento da distribuição de nêutrons, ou seja, a equação de transporte é a descrição fundamental e exata da população neutrônica de um reator.

A solução desta equação é muito complicada, mesmo para problemas simplificados, principalmente por ser uma equação de 7 variáveis independentes,(i.e., x, y, z, $\varphi, \theta, E$ e t), e pela complexidade de dependência das seções de choque macroscópica em espaço e em energia. Além disso, em problemas físicos, somente as quantidades integrais são observáveis, e a equação gera detalhes mais do que suficientes para o cálculo de parâmetros que descrevem o sistema, e.g., taxa de reação, potência, etc.

Modelos simplificados foram desenvolvidos baseados em hipótese físicas adequadas, como por exemplo, o modelo de difusão e de multigrupo, para que a partir dos quais possam ser desenvolvidos algoritmos numéricos factíveis e precisos. A versão mais simples da teoria de transporte é a de difusão. Apesar das restrições da sua validade, a teoria de difusão fornece uma boa aproximação da solução de equação de transporte. A equação de difusão no modelo de multigrupo obtida a partir da equação de transporte é escrita como [40]: 


$$
\begin{gathered}
{\left[\frac{1}{v_{g}} \frac{\partial}{\partial t}-\nabla \cdot D_{g} \nabla+\Sigma_{t g}\right] \Phi_{g}(\boldsymbol{r}, t)=\left[\sum_{g^{\prime}=1}^{G} \Sigma_{g^{\prime} g}+\chi_{g_{g^{\prime}=1}}^{G}(1-\beta) v_{g^{\prime}}, \Sigma_{f g^{\prime}}\right] \Phi_{g^{\prime}}(\boldsymbol{r}, t)+S_{g}(\boldsymbol{r}, t),} \\
\left(g^{\prime}=1,2, \ldots, G\right) \\
{\left[\frac{\partial}{\partial t}+\lambda_{j}\right] C_{j}(\boldsymbol{r}, t)=\beta_{j}\left[\sum_{g^{\prime}=1}^{G} v_{g^{\prime}}, \Sigma_{f g^{\prime}} \Phi_{g^{\prime}}(\boldsymbol{r}, t)\right],}
\end{gathered}
$$

onde $\Phi_{g}(\boldsymbol{r}, t)$ e $C_{j}(\boldsymbol{r}, t)$ são o fluxo do grupo $g$ e a concentração de precursores da família $j$, respectivamente, e as demais constantes são definidas conforme as definições clássicas.

Existem algumas restrições na validade do modelo de difusão, tais como a isotropia do meio e a dependência fraca das seções de choque no espaço, ou seja, para meios altamente anisotrópicos e heterogêneos o modelo de difusão não é um modelo adequado. 


\subsection{A Cinética Pontual e os Parâmetros Cinéticos}

Considere um sistema multiplicativo em estado estacionário, ou seja, um sistema cuja dependência temporal é nula, e a equação de transporte descrevendo o sistema pode ser simbolicamente escrita como:

$$
L_{0} \Phi_{s}+M_{0} \Phi_{s}+S_{0}=0
$$

onde o subíndice $s$ indica que o fluxo é estacionário e o subíndice zero foi acrescentado nos operadores para indicar que são do sistema de referência. $O$ operador $M_{0}$ é a soma dos operadores de multiplicação pronta, $M_{p, 0}$ e atrasada, $M_{d, 0}$.

O sistema descrito pela Equação (2.4) é subcrítico, em estado estacionário acionado por uma fonte externa, $S_{0}$, e isso não deve ser confundido com sistema crítico. Um sistema crítico é aquele que atinge o estado estacionário sem auxílio de fonte externa, ou seja:

$$
L_{0} \Phi_{c r}+M_{0} \Phi_{c r}=0
$$

em outras palavras, "todo sistema crítico é estacionário, mas nem todo sistema estacionário é crítico".

A solução da Equação (2.5) (denominada "equação do reator") fornece a distribuição de nêutrons no sistema crítico $\left(K_{e f f}=1\right)$. Entretanto, para pesquisar se o sistema é subcrítico, crítico, ou supercrítico deve se solucionar o problema de autovalor:

$$
L_{0} \varphi=-\frac{1}{K_{e f f}} M_{0} \varphi
$$

onde $1 / K_{\text {eff }}$ é o autovalor, e $\varphi$ a autofunção. Se $K_{\text {eff }}$ é 1, então, a Equação (2.6) reduz-se à Equação (2.5), $\varphi$ é o fluxo crítico. 
Se integrar a Equação (2.6) em todo espaço da fase (discussão sobre a integração em espaço da fase será feita em breve), i. e.:

$$
\left\langle L_{0} \Phi_{c r}\right\rangle=-\frac{1}{K_{e f f}}\left\langle M_{0} \Phi_{c r}\right\rangle
$$

que indica claramente a definição física de $K_{\text {eff }}$ que é produção global dividido pela destruição global, ou seja:

$$
K_{e f f}=-\frac{\left\langle M_{0} \Phi_{c r}\right\rangle}{\left\langle L_{0} \Phi_{c r}\right\rangle}
$$

Em sistemas críticos, como estabelecido por Henry [41] e Keepin [25], nos primórdios da cinética de reatores nos anos 60, após uma separação do fluxo angular nas variáveis $\boldsymbol{p}$ do espaço de fase $(r, \boldsymbol{\Omega}, E)$ e $t$, em uma função amplitude $P(t)$ e uma função forma $\varphi(\boldsymbol{p}, t)$ [fatorização], i.e.:

$$
\Phi=\varphi(\boldsymbol{p}, t) \cdot P(t)
$$

e assumindo a não dependência temporal da função forma e a homogeneidade do meio:

$$
\Phi(r, t)=\Phi_{c r}(r) \cdot P(t)
$$

as clássicas equações de cinética pontual podem ser obtidas [41], após a multiplicação das Equações (2.1.a) e (2.1.b) por uma função adjunta, $\Phi_{s}{ }^{+}$que é a solução da equação adjunta da (2.5) já que o sistema de referência, agora, é crítico:

$$
L_{0}^{+} \Phi_{c r}^{+}+M_{0}^{+} \Phi_{c r}^{+}=0
$$

$L_{0}{ }^{+}$e $M_{0}{ }^{+}$são os operadores adjuntos hermitianos de $L_{0}$ e $M_{0}$ (a discussão sobre os 
operadores adjuntos será feita no Apêndice 2), respectivamente, ou seja,

$$
\left\langle\Phi_{c r}, M_{0} \Phi_{c r}\right\rangle=\left\langle M_{0}^{+} \Phi_{c r}, \Phi_{c r}\right\rangle \text { e }\left\langle\Phi_{c r}, L_{0} \Phi_{c r}\right\rangle=\left\langle L_{0}^{+} \Phi_{c r}, \Phi_{c r}\right\rangle ;
$$

onde $\langle f, g\rangle$ é o produto interno das funções $f$ e $g$ no espaço da fase, i.e.:

$$
\langle f, g\rangle=\iiint f^{+} \cdot g d \Omega d E d V
$$

Considerando, sem perda de generalidade*, apenas 1 família de precursores e sem fonte externa, e assumindo perturbações nos operadores $L_{0}$ ou $M_{0}$ (e.g. $L=L_{0}+\delta L, M=M_{0}+\delta M$ ), e a teoria da perturbação de primeira ordem (i.e. $\delta^{2} \sim 0$ ), obtém-se:

$$
\frac{1}{v} \frac{\partial \Phi}{\partial t}=\left(L_{0}+\delta L\right) \Phi+\left(M_{p, 0}+\delta M_{p}\right) \Phi+\lambda C
$$

$\mathrm{e}$

$$
\frac{\partial C}{\partial t}=\left(M_{d, 0}+\delta M_{d}\right) \Phi-\lambda C
$$

O termo da perturbação no operador multiplicação atrasada, $\delta M_{d}$ pode ser desprezado, pois $M_{p} \gg M_{d}$ e apena pequenas perturbações estão sendo consideradas neste trabalho.

Agora, aplicando a fatorização (2.8) e a relação:

$$
L_{0}=-M_{0}=-\left(M_{p, 0}+M_{d, 0}\right)
$$

deduzida a partir da Equação (2.5), obtém-se:

$$
\frac{1}{v} \Phi_{c r} \frac{d P}{d t}=-M_{d, 0} \Phi_{c r} P+\left(\delta L+\delta M_{p}\right) \Phi_{c r} P+\lambda C
$$

$\mathrm{e}$

\footnotetext{
* Obviamente deveria considerar 6 famílias de precursores, mas, como o objetivo deste trabalho é teórico e visa apenas demonstrar a idéia da dependência dos parâmetros cinéticos com a subcriticalidade, será considerada somente 1 família de precursores, sem perda da generalidade.
} 


$$
\frac{d C}{d t}=\left(M_{d, 0}+\delta M_{d}\right) \Phi_{c r} P-\lambda C
$$

Multiplicando as duas equações por $\Phi_{c r}{ }^{+}$, e integrando em todo espaço, obtém-se as equações da cinética pontual:

$$
\frac{d P}{d t}=\frac{\left(\rho-\beta_{e f f}\right)}{\Lambda} P(t)+\lambda e,
$$

e

$$
\frac{d e}{d t}=\frac{\beta_{e f f} P(t)}{\Lambda}-\lambda e
$$

onde os parâmetros cinéticos $\rho$ (reatividade), $\beta_{\text {eff }}, \Lambda$, possuem uma definição consistente em termos da solução da equação adjunta para o sistema em estado estacionário perto da criticalidade $\left(K_{\text {eff }} \approx 1\right)$, ou seja [42]:

$$
\begin{aligned}
& \rho=\frac{1}{F}\left\langle\Phi_{c r}^{+},(\delta L+\delta M) \Phi_{c r}\right\rangle=\frac{\delta K_{e f f}}{K_{e f f}}, \\
& \beta_{e f f}=\frac{1}{F}\left\langle\Phi_{c r}^{+}, M_{d} \Phi_{c r}\right\rangle, \\
& \Lambda=\frac{1}{F}\left\langle\left(\frac{1}{v}\right) \Phi_{c r}^{+}, \Phi_{c r}\right\rangle, \\
& C=\frac{1}{\Lambda F}\left\langle\Phi_{c r}, C\right\rangle,
\end{aligned}
$$

com a normalização,

$$
F=\left\langle M_{p} \Phi_{c r}\right\rangle
$$


Desta forma num sistema crítico, os parâmetros cinéticos e a reatividade têm uma definição consistente, constituindo-se em parâmetros globais e efetivos. Assim a reatividade,

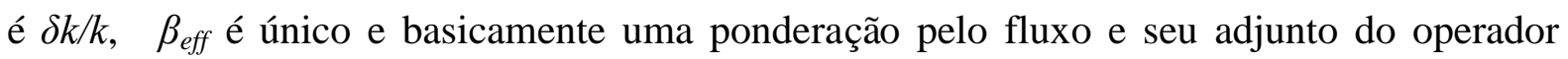
multiplicação atrasada pelo operador multiplicação pronta em todo espaço de fases, e $\Lambda$ uma ponderação do tempo médio de geração de nêutrons $\left(1 / \Sigma_{a} v\right)$. Ou seja, num sistema crítico, ou perto da criticalidade, os parâmetros cinéticos são únicos, e são valores integrais que para perturbações uniformes, permitem a solução das Equações (2.5) da cinética pontual para a descrição temporal do sistema.

Por outro lado, se a perturbação for localizada, a função forma tem dependência temporal, e o método "quase-estático" é adequado para a descrição temporal do sistema. Neste método se assume uma variação suave desta função que é recalculada numa escala de tempo maior do que as equações do tipo pontual em que a função amplitude é calculada [33].

O cálculo dos parâmetros cinéticos pode então ser facilmente obtido, da solução da equação do reator crítico e da sua adjunta, através, por exemplo, de códigos determinísticos (transporte ou difusão), e a utilização das definições destes parâmetros através das Equações (2.13).

Entretanto, conforme apresentado por Dulla a definição dos parâmetros cinéticos para sistemas subcríticos acionados por fontes externas, necessita um melhor embasamento teórico, e não é correto extrapolar diretamente o cálculo destes, a partir da metodologia utilizada em sistemas críticos.

A solução adjunta para o sistemas subcríticos acionados por fonte, em estado estacionário é dada pela equação adjunta da Equação (2.4):

$$
L_{0}^{+} \Phi_{s}^{+}+M_{0}^{+} \Phi_{s}^{+}+S_{0}^{+}=0
$$


Utilizando-se as mesmas técnicas de fatorização, de perturbação e de projeção discutidas para sistemas críticos, e desprezando o termo $\delta M_{d}$, obtém-se as seguinte expressões:

$$
\begin{aligned}
\frac{\left\langle\Phi_{s}^{+} \mid\left(\frac{1}{v}\right) \Phi_{s}\right\rangle}{I} \frac{d P}{d t}= & {\left[\frac{\left\langle\Phi_{s}^{+} \mid\left(\delta L+\delta M_{p}\right) \Phi_{s}\right\rangle}{I}-\frac{\left.\left.\left\langle\Phi_{s}^{+}\right|\left(\frac{1}{v}\right) \Phi_{s}\right)\right\rangle}{I}-\frac{\left\langle\Phi_{s}^{+} \mid S_{0}\right\rangle}{I}\right] P(t) } \\
& +\lambda \frac{\left\langle\Phi_{s}^{+} \mid C\right\rangle}{I}+\frac{\left\langle\Phi_{s}^{+} \mid S\right\rangle}{I}
\end{aligned}
$$

Desta forma, Dulla demonstra que equações do tipo cinética pontual para a função amplitude podem ser obtidas com uma consistente definição dos parâmetros cinéticos, ou seja [16]:

$$
\frac{d P(x)}{d t}=\frac{\left(\rho_{s}-\beta_{s}\right)}{\Lambda_{s}} P(t)+\lambda \mathbb{C}_{s}+\mathbb{S},
$$

$\mathrm{e}$

$$
\frac{\partial \mathbb{C}_{s}}{\partial t}=\beta_{s} P(t)-\lambda \mathbb{C}_{s},
$$

com

$$
\begin{aligned}
& \rho_{s}=\tilde{\rho_{s}}+\rho_{s, 0}, \\
& \tilde{\rho_{s}}=\frac{\left\langle\Phi_{s}^{+} \mid(\delta L+\delta M) \Phi_{s}\right\rangle}{I}, \\
& \rho_{s, 0}=-\frac{\left\langle\Phi_{s}^{+} \mid S_{0}\right\rangle}{I},
\end{aligned}
$$




$$
\beta_{S}=\frac{\left\langle\Phi_{s}^{+} \mid M_{d, 0} \Phi_{s}\right\rangle}{I},
$$

$$
\Lambda_{s}=\frac{\left\langle\Phi_{s}^{+} \mid\left(\frac{1}{v}\right) \Phi_{s}\right\rangle}{I},
$$

$$
\mathbb{S} \equiv \frac{\left\langle\Phi_{s}^{+} \mid S\right\rangle}{I},
$$

$\mathrm{e}$

$$
\mathbb{C} \equiv \frac{\left\langle\Phi_{s}^{+} \mid C\right\rangle}{I}
$$

onde o parâmetro I sugerido por Dulla, está relacionado com a "importância" da primeira geração de nêutrons introduzida no sistema por fissões e nêutrons da fonte, ou seja [16]:

$$
I \equiv\left\langle\Phi_{s}^{+} \mid M_{0} \Phi_{s}\right\rangle+\left\langle\Phi_{s} \mid S_{0}\right\rangle
$$

O "formalismo de Dulla" permite uma interpretação da reatividade de fonte, com o chamado "Fator de Multiplicação de fonte" $\left(k_{S}\right)$ :

$$
k_{s}=\frac{\left\langle M \Phi_{s}\right\rangle}{\left\langle M \Phi_{s}+S\right\rangle} \text {. }
$$

Para a obtenção da relação entre a reatividade generalizada $\left(\rho_{s}\right) \operatorname{com} k_{S}$, Dulla utiliza a técnica de projeção com a função adjunta dada pela solução da equação (2.18) para obter,

$$
\tilde{\rho_{s}}=\frac{\delta k_{s}}{k_{s}} \frac{\left\langle M_{0} \Phi_{s}\right\rangle}{I},
$$




$$
\frac{\delta k_{s}}{k_{s}}=\frac{\left\langle S_{0}\right\rangle}{\left\langle M_{0} \Phi_{s}\right\rangle+\left\langle S_{0}\right\rangle}\left[\frac{\left\langle\Phi_{s}^{+} \mid(\delta L+\delta M) \Phi_{s}\right\rangle}{\left\langle M_{0} \Phi_{s}\right\rangle}-\frac{\langle\delta S\rangle}{\left\langle S_{0}\right\rangle}\right],
$$

desprezando-se perturbação devido ao termo da fonte relativamente a outras perturbações:

$$
\frac{\delta k_{s}}{k_{s}} \approx \frac{\left\langle\Phi_{s}^{+} \mid(\delta L+\delta M) \Phi_{s}\right\rangle}{\left\langle M_{0} \Phi_{s}\right\rangle}
$$

Mais além, Dulla mostra a consistência de seu formalismo, quando o sistema aproxima-se da criticalidade, ou seja, $K_{\text {eff }} \rightarrow 1$. 


\subsection{Problemas Resolvidos}

Como foi visto na seção anterior, para poder calcular os parâmetros cinéticos é necessário obter previamente a função de fluxo estacionário e sua adjunta que são soluções da equação estacionária e a sua adjunta, respectivamente. Estas equações serão resolvidas, inicialmente, usando o modelo de 1 grupo, e depois, de 2 grupos de energia. Os problemas apresentados aqui são problemas ideais cujas constantes físicas estão disponíveis na literatura.

Note que, o sistema a ser tratado aqui é de potência zero, ou seja, no problema não será considerada a realimentação do sistema.

O problema de um sistema de geometria plana de largura $H$, meio homogêneo é um problema unidimensional. Será assumida a isotropia de espalhamento e fluxo nulo na fronteira como condição de contorno, e conforme sugerido por Dulla $\mathrm{S}_{0}{ }^{+}$é escolhido como sendo $v \Sigma_{f}$.

\subsubsection{Modelo de 1 grupo de energia}

No modelo de difusão para 1 grupo de energia os operadores da Equação (2.4) são escritos na seguinte forma:

$$
L_{0} \equiv\left(D \frac{\partial^{2}}{\partial x^{2}}-\Sigma_{a, 0}\right) \ldots,
$$

e

$$
M_{0} \equiv v \Sigma_{f, 0} \cdots \cdot
$$


Para solucionar a equação será utilizado o método de expansão, que consiste em expandir uma função, neste caso o fluxo estacionário, $\Phi_{s}(x)$, em autofunções base do problema de autovalores. Sabe-se que as soluções da Equação de Helmholtz formam uma base completa. A Equação de Helmholtz em uma dimensão é:

$$
\frac{d \varphi_{n}(x)}{d x}=-B_{n}^{2} \varphi_{n}(x)
$$

com condição de contorno:

$$
\varphi_{n}\left( \pm \frac{H}{2}\right)=0
$$

onde $B_{n}$ é o autovalor da função $\Phi_{n}(x)$. A solução da Equação (2.28) é facilmente obtida:

$$
\varphi_{n}(x)=\cos \left(B_{n} x\right)
$$

e os autovalores que satisfazem a condição de contorno são:

$$
B_{n}=\frac{(2 n+1)}{H} \pi, \quad(n=0,1,2, \ldots)
$$

Portanto,

$$
\varphi_{n}(x)=\cos \left(\frac{(2 n+1)}{H} \pi x\right)
$$

É possível expandir tanto o fluxo estacionário como a fonte externa em termo das autofunções:

$$
\Phi_{s}=\sum_{n=0}^{\infty} A_{n} \cdot \varphi_{n}
$$


e

$$
S_{0}=\sum_{n=0}^{\infty} S_{n} \cdot \varphi_{n},
$$

onde os $A_{n}$ e $s_{n}$ são coeficientes a serem determinados.

Substituindo (2.27) e (2.33) na Equação(2.4), obtém-se a relação entre $A_{n}$ e $\mathrm{s}_{n}$, sendo:

$$
A_{n}=\frac{s_{n}}{D B_{n}^{2}+\Sigma_{a, 0}-v \Sigma_{f, 0}}=\frac{s_{n}}{D\left(B_{n}^{2}+B_{m}^{2}\right)},
$$

onde

$$
B_{m}^{2} \equiv \frac{\left(v \Sigma_{f, 0}-\Sigma_{a, 0}\right)}{D},
$$

que é definido como "buckling" material.

Os $s_{n}$ são obtidos utilizando-se a propriedade de ortogonalidade das autofunções da equação de Helmholtz. Portanto, multiplica-se ambos lados da Expressão (2.33.b) por $\varphi_{m}(x)$, onde $m \geq 0$, um número integral qualquer; e integra-se ambos lados em todo sistema:

$$
\int_{-\frac{H}{2}}^{\frac{H}{2}} \varphi_{m}(x) S_{0}(x) d x=\int_{-\frac{H}{2}}^{\frac{H}{2}} \varphi_{m}(x) \sum_{n=0}^{\infty} S_{n} \varphi_{n}(x) d x,
$$

Devido à ortogonalidade, o produto de $\varphi_{m}(x)$ e $\varphi_{n}(x)$ é diferente de zero se e somente se $m$ for igual a $n$. Portanto: 


$$
S_{n}=\frac{\int_{-\frac{H}{2}}^{\frac{H}{2}} \varphi_{n}(x) S_{0}(x) d x}{\int_{-\frac{H}{2}}^{\frac{H}{2}} \varphi_{n}^{2}(x) d x}=\frac{2}{H} \int_{-\frac{H}{2}}^{\frac{H}{2}} \varphi_{n}(x) S_{0}(x) d x,
$$

e, finalmente,

$$
s_{n}=\frac{2}{H} \int_{-\frac{H}{2}}^{\frac{H}{2}} \varphi_{n}(x) \cos \left(B_{n} x\right) d x
$$

Substituindo (2.34) e (2.36.c) em (2.33.a), a expressão do $\Phi_{s}(x)$ é obtida:

$$
\Phi_{S}(x)=\left(\frac{2}{H}\right) \sum_{n=0}^{\infty}\left\{\frac{\cos \left(B_{n} x\right)}{D\left(B_{n}^{2}+B_{m}^{2}\right)} \int_{-\frac{H}{2}}^{\frac{H}{2}} S_{0}(x) \cos \left(B_{n} x\right) d x\right\}
$$

Note que no problema de 1 grupo, os operadores $L_{0}$ e $M_{0}$ são idênticos aos seus adjuntos, $\mathrm{L}_{0}{ }^{+}$e $\mathrm{M}_{0}{ }^{+}$, respectivamente, ou seja, são hermitianos. Sendo assim, o fluxo adjunto pode ser determinado diretamente da Expressão (2.37), substituindo $S_{0}(x)$ por $S_{0}^{+}(x)$, ou seja, por $v \Sigma_{f}$,

$$
\Phi_{s}^{+}(x)=\left(\frac{2}{H}\right) \sum_{n=0}^{\infty}\left\{\frac{\cos \left(B_{n} x\right)}{D\left(B_{n}^{2}+B_{m}^{2}\right)} \int_{-\frac{H}{2}}^{\frac{H}{2}} S_{0}^{+}(x) \cos \left(B_{n} x\right) d x\right\} \text {. }
$$

Para o caso dos sistemas críticos, as soluções das Equações (2.5) e (2.10) são a própria autofunção do problema. Ou seja:

$$
\Phi_{c r}(x)=\Phi_{c r}^{+}(x)=A \cos \left(\frac{\pi x}{H}\right)
$$

onde $A$ é um coeficiente determinado dado o nível da potência do reator: 


$$
P=\int_{-\frac{H}{2}}^{\frac{H}{2}} \Sigma_{f} \Phi_{c r}(x) d x
$$

A Figura 2 mostra os gráficos dos fluxos calculados para um sistema subcrítico acionado por uma fonte igual ao delta de Dirac: $S_{0}(x)=\delta(x)$. Como já foi mencionado na seção anterior, trata-se de um reator homogêneo tipo placa de largura $H$. Os dados nucleares utilizados no cálculo encontram-se na Tabela 2; são os mesmos utilizados no trabalho de mestrado de Fernanado Oliveira [37] e são de um sistema rápido típico; a largura $H=$ 23,13343834 cm, foi calculada para $K_{\text {eff }}=0,98$, para sistema subcrítico, e $H=23,36830160$ $\mathrm{cm}$, para sistema crítico, ou seja, para $K_{\text {eff }}=1$. O fator de multiplicação $K_{\text {eff }}$ é defindo como [40]:

$$
K_{e f f}=\frac{\frac{v \Sigma_{f}}{\Sigma_{a}}}{1+L^{2} B_{g}^{2}},
$$

onde $B_{g}{ }^{2}$ é "buckling" geométrico, que é uma medida da curvatura de fluxo; neste caso é $(\pi / H) ; \quad L$ é o comprimento de difusão dos nêutrons.

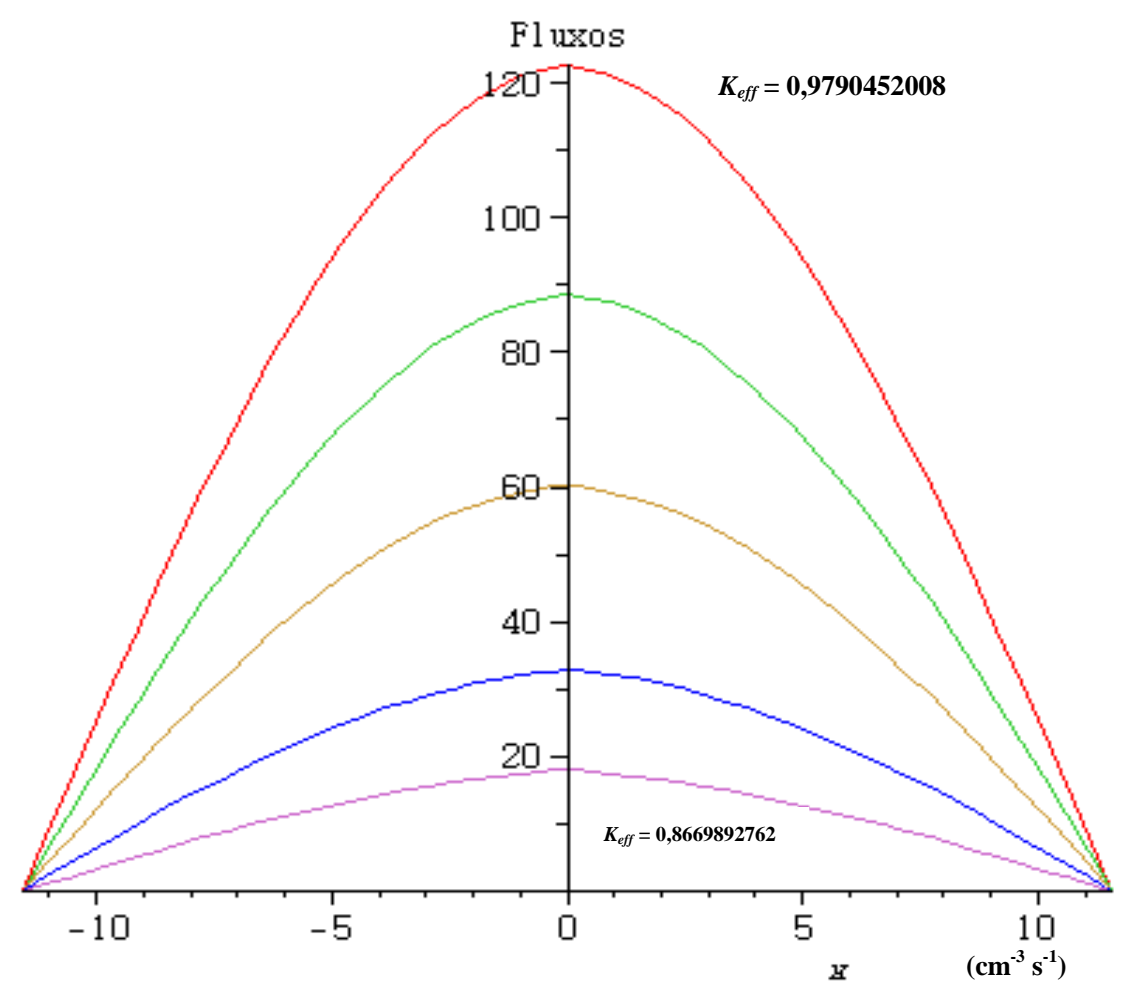

Figura 2: Fluxos para vários valores de $K_{\text {eff }}(0,9790452008 \sim 0,8669892762)$ 


\begin{tabular}{|c|l|}
\hline $\mathbf{1} / \boldsymbol{v}\left[\mathrm{s} \mathrm{cm}^{-1}\right]$ & $0,9062 \mathrm{e}-7$ \\
\hline $\boldsymbol{\Sigma}_{\boldsymbol{t}}\left[\mathrm{cm}^{-1}\right]$ & $3,45987 \mathrm{e}-1$ \\
\hline $\boldsymbol{\Sigma}_{\boldsymbol{a}}\left[\mathrm{cm}^{-1}\right]$ & $1,58430 \mathrm{e}-2$ \\
\hline $\boldsymbol{\nu} \boldsymbol{\Sigma}_{f}\left[\mathrm{~cm}^{-1}\right]$ & $3,33029 \mathrm{e}-2$ \\
\hline $\boldsymbol{\Sigma}_{\boldsymbol{s}}\left[\mathrm{cm}^{-1}\right]$ & $3,30178 \mathrm{e}-1$ \\
\hline
\end{tabular}

Tabela 2. Dados nucleares de um sistema rápido utilizados para o modelo de 1 grupo [37].

A Figura 3 mostra um gráfico de valores da integral dos fluxos em função de $K_{\text {eff. }}$. Note que a intensidade do fluxo aumenta com $K_{\text {eff. }}$

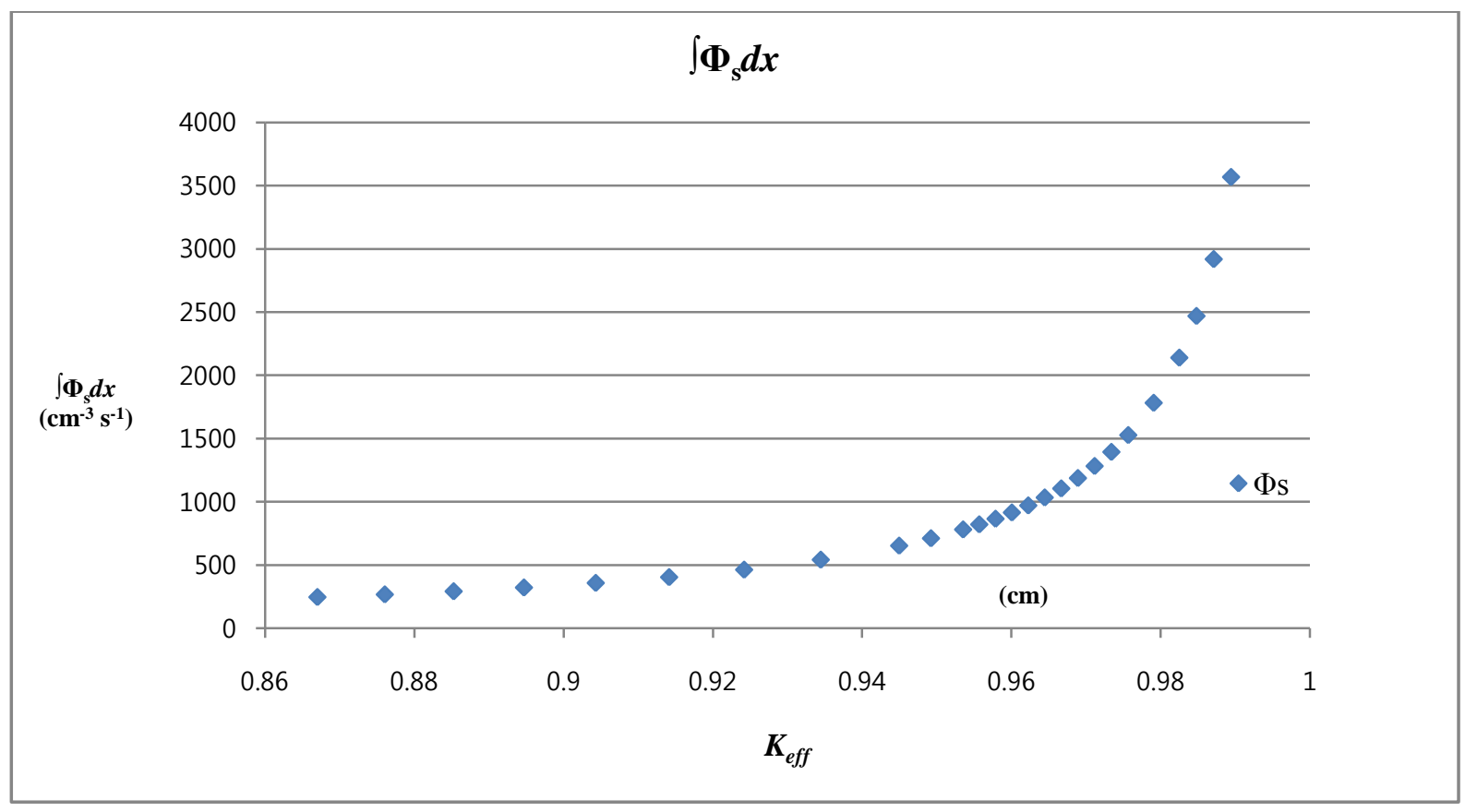

Figura 3. Integrais dos fluxos em função de $K_{\text {eff. }}$.

O coeficiente de difusão $D$ foi calculado usando-se a seguinte definição [44]:

$$
D=\frac{1}{3 \Sigma_{t}} \quad
$$

Utilizando as expressões dos fluxos e a Definição (2.42), e os dados da Tabela 2, foram obtidos os valores numéricos dos parâmetros cinéticos, ou seja, para o sistema crítico, 
usando as Equações clássicas (2.17), e para o sistema subcrítico acionado por fonte, com o uso das novas Equações (2.21). Através desses resultados pode-se estudar o comportamento

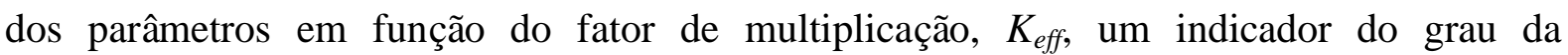
subcriticalidade dos sistemas multiplicativos.

Existem várias maneiras de alterar o grau da subcriticalidade dos sistemas. Aqui optou-se pela maneira mais simples: variando-se o valor da seção de choque, $\Sigma_{a}$, sem alterar a geometria. Para cada valor da seção de choque de absorção terá um valor de $K_{\text {eff }}$ e de fluxos $\Phi_{s}$ e $\Phi_{s}{ }^{+}$. Ou seja, o valor de $\Sigma_{a}$ determina o estado de cada sistema.

Diferente da definição clássica, a nova definição da reatividade contém um termo de perturbação, $\delta L$ ou $\delta M$. Aqui cabe uma observação: não se deve confundir a variação do estado de um sistema com a perturbação efetuada no sistema. Neste trabalho será assumido que, as variações e as perturbações sejam efetuadas apenas na seção de choque de absorção. Portanto, recomenda-se que o leitor entenda claramente a diferença entre esses dois conceitos antes de prosseguir. Como já foi mencionado, para cada valor de $\Sigma_{a}$ corresponde um estado do sistema, e cada estado pode ser perturbado como conseqüência da perturbação em $\Sigma_{a}$. Porém, é de se notar que, como essas perturbações são pequenas, o estado do sistema (fluxo) não se altera. Neste trabalho, foi adotada perturbação de $2 \%$ para cada $\Sigma_{a}$.

Os valores dos parâmetros cinéticos calculados encontram-se na Tabela A.1. do Apêndice 1. Nas Figuras 4, 5 e 6, pode-se ver as diferenças de valores dos parâmetros $(\beta, \Lambda$ e $\rho$, respectivamente) obtidos para cada método.

O coeficiente de difusão, $D$, foi mantido como constante durante todo o cálculo. 


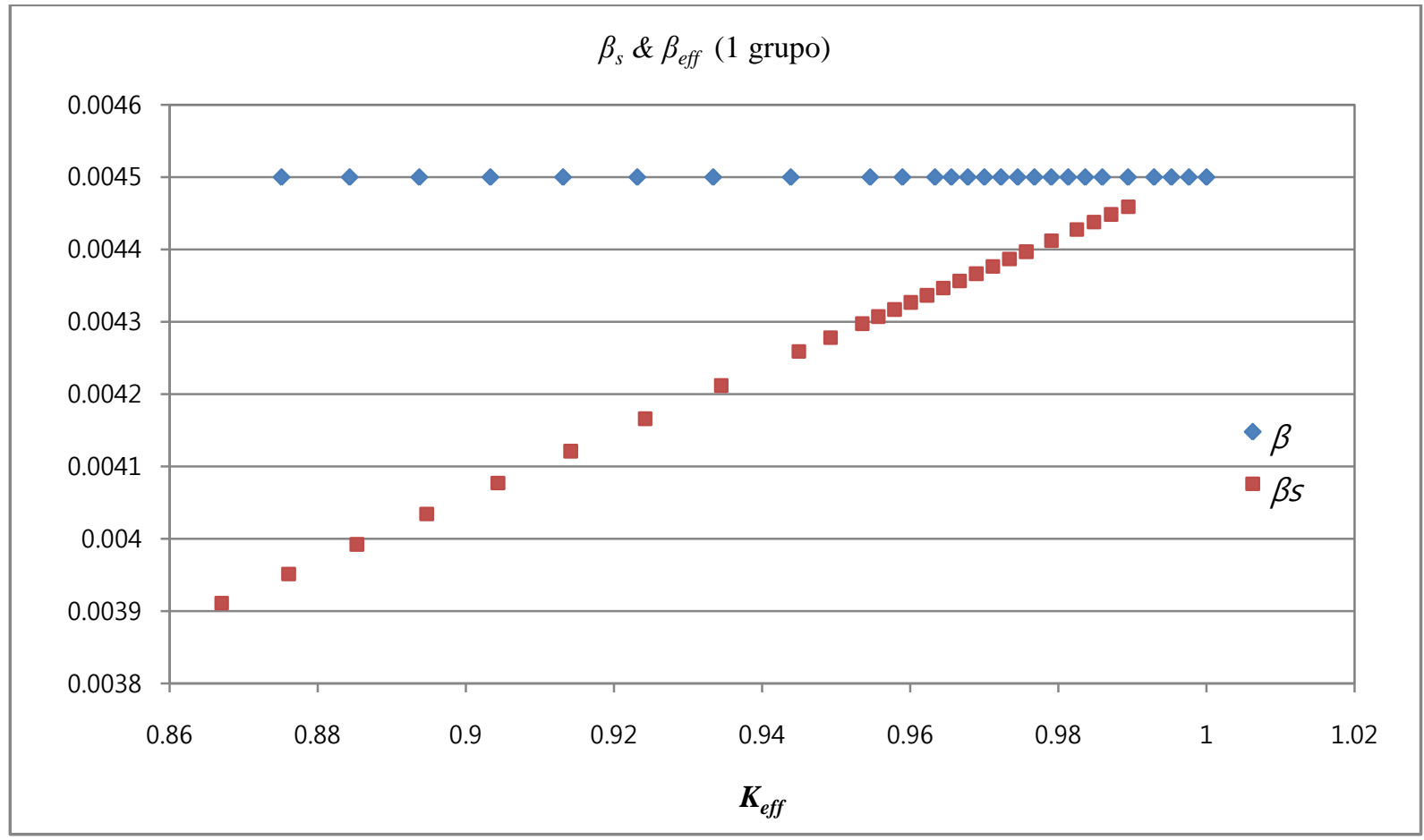

Figura 4. Os valores dos $\beta_{\text {eff }}$ e $\beta_{s}$ em função do $K_{\text {eff }}$ no modelo de 1 grupo.

Pode-se observar na Figura 4, que os $\beta$ calculados pelo método clássico se mantem praticamente constantes, enquanto os resultados obtidos variam com $K_{\text {eff }}$, e que os valores se aproximam na região em que $K_{\text {eff }}$ tende a 1, i.e., perto da criticalidade.

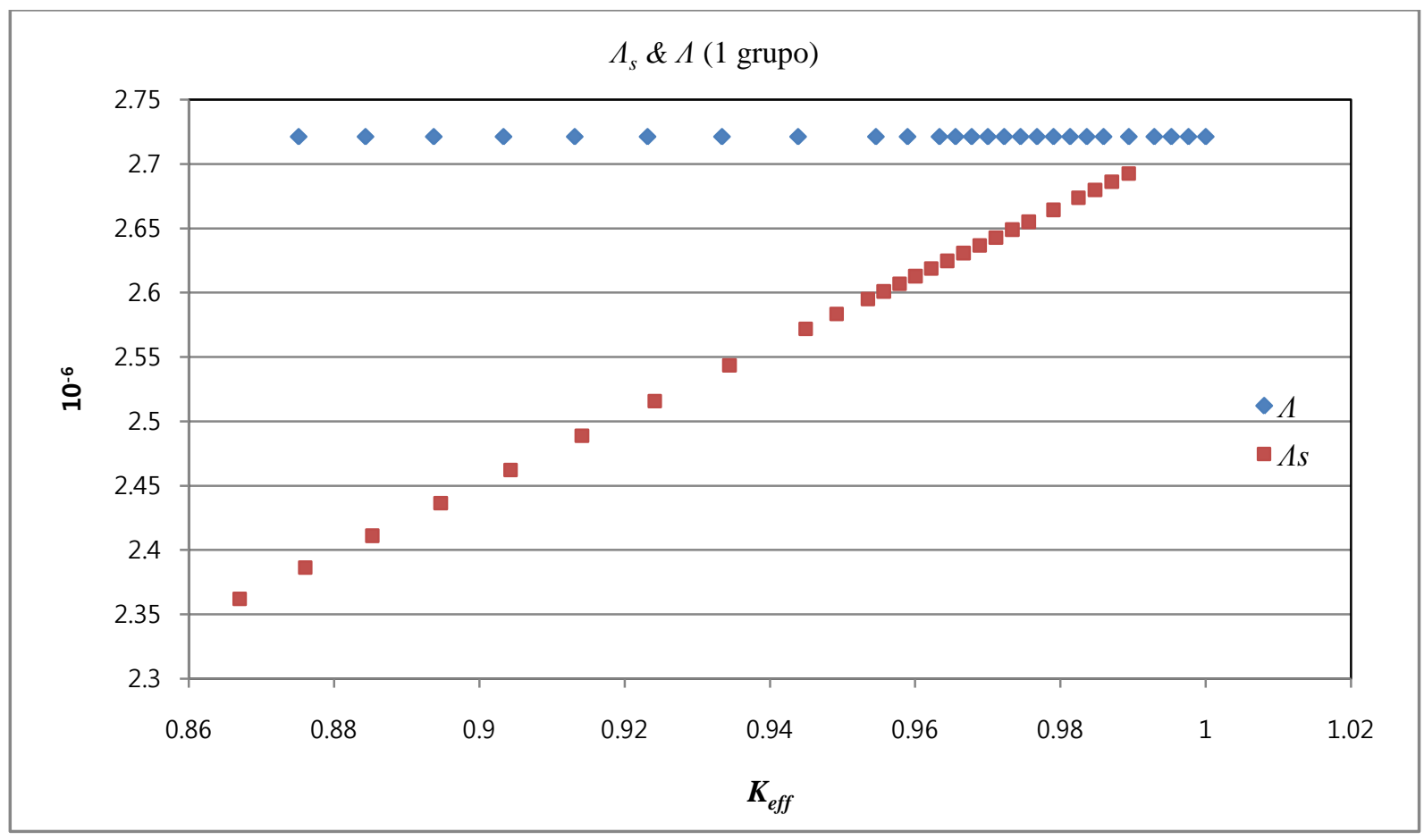

Figura 5. Os valores dos $\Lambda$ e $\Lambda_{s}$ em função do $K_{\text {eff }}$ no modelo de 1 grupo. 
Como no caso de $\beta$, na Figura 5 pode ser observado que os $\Lambda$ obtidos pelo método clássico sofrem variações insignificantes, ao passo que os $\Lambda_{s}$ aumentam com $K_{\text {eff. }}$, e os ambos resultados se aproximam perto da criticalidade $\left(K_{\text {eff: }}=1\right)$. Este fato demonstra que utilizar-se para estes parâmetros valores calculados na criticalidade nas equações da cinética pontual leva a resultados incorretos.

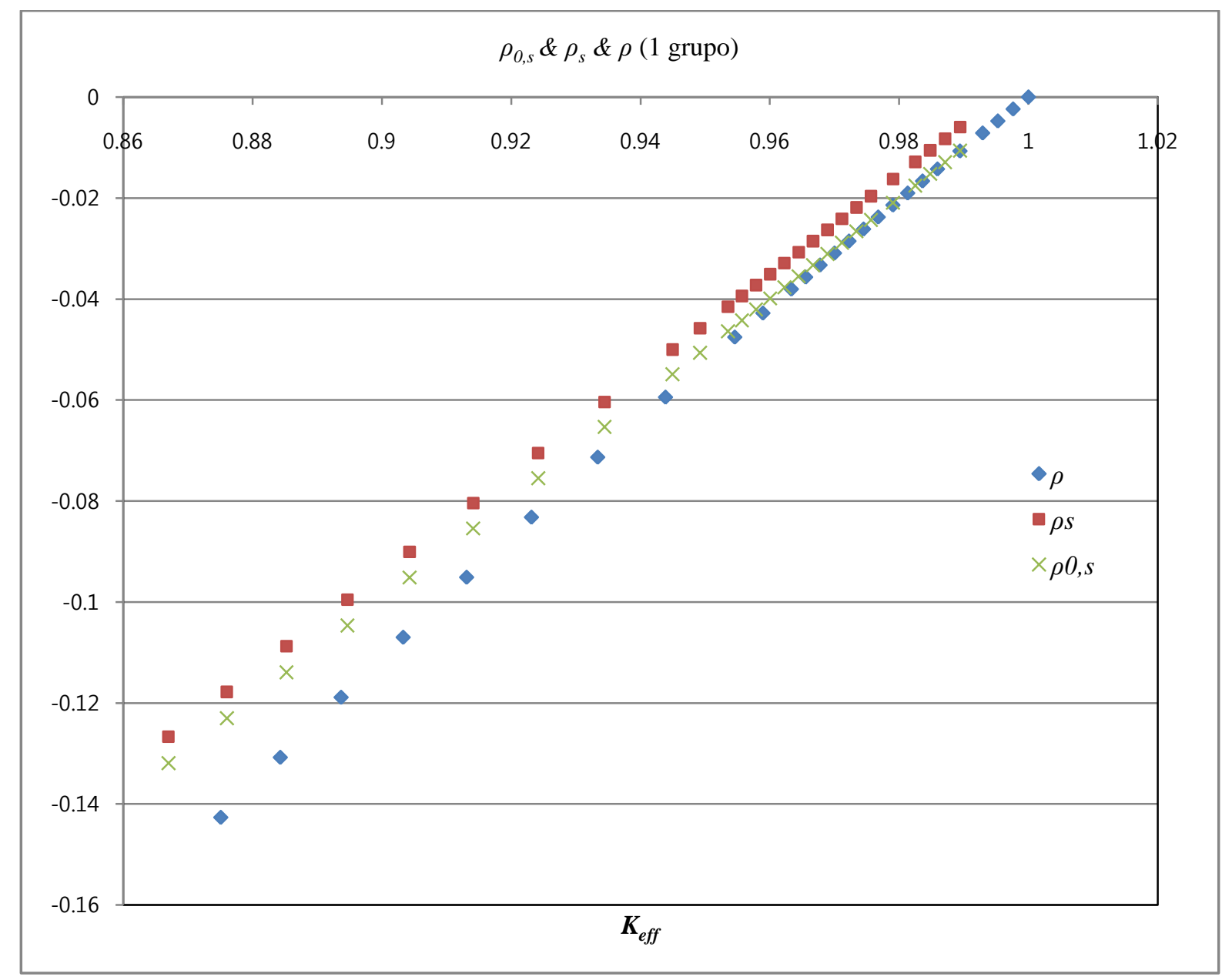

Figuara 6. Os valores dos $\rho, \rho_{s, 0}$ e $\rho_{s}$ em função dos $K_{\text {eff }}$ no modelo de 1 grupo.

Os $\rho_{s, 0}$ e $\rho_{s}$ da Figura 6 são valores para o sistema inicial e perturbado, respectivamente, e os $\rho$ são calculados pelo método clássico. Como resultado da perturbação equivalente ao acréscimo de $2 \%$ da seção de choque de absorção em cada grau da subcriticalidade, os $\rho_{s}$ se tornam menos negativos que os $\rho_{s, 0}$. Pode-se observar que os três parâmetros variam com $K_{\text {eff. }}$, mas que as diferenças entre os parâmetros diminuem quando $K_{\text {eff }}$ tende a 1 . 


\subsubsection{Modelo de 2 grupos de energia}

É óbvio que o modelo de difusão em 1 grupo de energia não é, em geral, adequado para descrever um sistema multiplicativo satisfatoriamente. A principal deficiência do modelo está na suposição de que todos os nêutrons possam ser caracterizados por uma única rapidez ou energia, embora o espectro de energia dos nêutrons se estenda de $0,01 \mathrm{eV}$ até 10 $\mathrm{MeV}$ [40]. Além disso, sabe-se que as seções de choques de reações entre núcleo e nêutrons dependem sensivelmente da energia dos nêutrons. Porém, pode-se afirmar que, com uma judiciosa escolha das seções de choque, o modelo de 1 grupo funciona razoavelmente.

O modelo de 2 grupos de energia não pode ser muito melhor que o de 1 grupo para descrever um sistema multiplicativo, mas, não há dúvida de que os resultados obtidos usando modelo de 2 grupos sejam mais próximos à da realidade. Deve se notar, que no modelo de 1 grupo, os operadores são idênticos aos seus adjuntos, e que, porém, a partir do modelo de 2 grupos isso não acontece.

O problema de 2 grupos de energia consiste em dois problemas de 1 grupo, porém, acoplados entre si devido à variação dos nêutrons em energia. O grupo de maior energia será denomido de grupo rápido, e o de menor energia, de grupo térmico. É comum indexar um número menor ao grupo de energia superior. Desta forma, o grupo 1 vai ser o rápido, e o grupo 2, o térmico.

Neste trabalho serão assumidas as seguintes hipóteses para simplificar o problema de 2 grupos:

a) não ocorre "up-scattering", ou seja, os nêutrons não são transportados ao nível superior de energia por espalhamento;

b) as fissões ocorrem somente no grupo térmico, ou seja, no grupo 2;

c) todos os nêutrons de fissão, nascem no grupo rápido, i.e., no grupo 1; e

d) todos os nêutrons provenientes da fonte externa nascem no grupo rápido. 
Como foi mencionado previamente, o problema de 2 grupos consiste em um sistema de duas equações acopladas. No estado estacionário essas equações têm as seguintes formas:

$$
\begin{aligned}
& \frac{D_{1} d^{2} \Phi_{s 1}}{d x^{2}}-\Sigma_{R 1} \Phi_{s 1}+v_{2} \Sigma_{f 2} \Phi_{s 2}+S_{1}=0 \\
& \frac{D_{2} d^{2} \Phi_{s 2}}{d x^{2}}-\Sigma_{a 2} \Phi_{s 2}+\Sigma_{s 12} \Phi_{s 1}=0
\end{aligned}
$$

onde $\Sigma_{R 1}$ é a seção de choque de remoção ou "removal" do grupo 1, e $\Sigma_{s 12}$ de espalhamento do grupo 1 ao grupo 2. As Equações (2.43) podem ser escritas em forma matricial:

$$
\left(\underline{L}_{0}+\underline{M}_{0}\right) \Phi_{s}+\boldsymbol{S}_{0}=0
$$

onde

$$
\begin{aligned}
& \underline{L}_{0} \equiv\left(\begin{array}{cc}
\frac{D_{1} d^{2}}{d x^{2}}-\Sigma_{R 1} & 0 \\
\Sigma_{s 12} & \frac{D_{2} d^{2}}{d x^{2}}-\Sigma_{a 2}
\end{array}\right), \\
& \underline{M}_{0} \equiv\left(\begin{array}{cc}
0 & v_{2} \Sigma_{f 2} \\
0 & 0
\end{array}\right], \\
& \boldsymbol{\Phi}_{\boldsymbol{n}}=\left[\begin{array}{c}
\Phi_{n} \\
\boldsymbol{\Phi}_{n}
\end{array}\right] \quad \text { e } \quad \boldsymbol{S}_{\boldsymbol{0}}=\left[\begin{array}{c}
S_{1} \\
0
\end{array}\right]
\end{aligned}
$$

Usando o método de expansão, como no caso de 1 grupo: 


$$
\boldsymbol{\Phi}_{\boldsymbol{s}}=\sum_{n=0}^{\infty} \underline{A}_{n} \cdot \boldsymbol{\varphi}_{\boldsymbol{n}}
$$

$\mathrm{e}$

$$
\boldsymbol{S}_{\boldsymbol{0}}=\sum_{n=0}^{\infty} \underline{\underline{S}}_{n} \cdot \boldsymbol{\varphi}_{\boldsymbol{n}}
$$

onde os $\underline{A}_{n}$ e $\underline{s}_{n}$ são, agora, matrizes diagonais cujos elementos são coeficientes das autofunções da equação de Helmholtz e $\varphi_{n}$ vetor dessas autofunções, ou seja:

$$
\varphi_{n}(x)=\cos \left(\frac{(2 n+1)}{H} \pi x\right) \text {. }
$$

Substituindo (2.46) na equação (2.44) obtém-se a relação entre $\underline{A}_{n}$ e $\underline{s}_{n}$ :

$$
\underline{A}_{n}=-\left(\underline{L}_{0}+\underline{M}_{0}\right)^{-1} \underline{s}_{n}
$$

Novamente, os $\underline{s}_{n}$ são determinados usando a propriedade de ortogonalidade das autofunções de Helmholtz, e a solução é dada como:

$$
\boldsymbol{\Phi}_{\boldsymbol{s}}=-\frac{2}{H} \sum_{n=0}^{\infty} \Phi_{n}\left[\int_{-\frac{H}{2}}^{\frac{H}{2}} \cos \left(\frac{\left(2 n_{1}+1\right) \pi}{H} x\right) S_{1} d x\right]\left(\underline{L}_{0}+\underline{M}_{0}\right)^{-1}\left[\begin{array}{l}
1 \\
0
\end{array}\right] \text {. }
$$

Não é difícil especular que a partir do problema de 2 grupos os operadores não são necessariamente idênticos aos seus adjuntos. Como todas as matrizes do problema contêm apenas elementos reais, as adjuntas são suas transpostas. Ou seja:

$$
\underline{L}_{0}^{+} \equiv\left(\begin{array}{cc}
\frac{D_{1} d^{2}}{d x^{2}}-\Sigma_{R 1} & \Sigma_{s 12} \\
0 & \frac{D_{2} d^{2}}{d x^{2}}-\Sigma_{a 2}
\end{array}\right),
$$




$$
\underline{M}_{0}{ }^{+} \equiv\left[\begin{array}{cc}
0 & 0 \\
v_{2} \Sigma_{f 2} & 0
\end{array}\right],
$$

e

$$
\boldsymbol{S}_{0}^{+}=\left[\begin{array}{c}
0 \\
v \Sigma_{f}
\end{array}\right]
$$

O procedimento para obter o fluxo adjunto é análodo ao anterior, e a solução é dada por:

$$
\boldsymbol{\Phi}_{\boldsymbol{s}}^{+}=-\frac{8 v_{2} \Sigma_{f}}{\pi H} \sum_{n=0}^{\infty} \Phi_{n} \frac{1}{(2 n+1)}\left(\underline{L}_{0}^{+}+\underline{M}_{0}^{+}\right)^{-1} \cdot\left[\begin{array}{l}
0 \\
1
\end{array}\right]
$$

O procedimento para resolver o problema de um sistema crítico está apresentada detalhadamente na Referência 44, e as soluções são:

$$
\begin{aligned}
& \Phi_{s 1}=\cos (\mu x)-\left(\frac{\cos \left(\frac{H}{2} \mu\right)}{\cosh \left(\frac{H}{2} \lambda\right)}\right) \cosh (\lambda x), \\
& \Phi_{s 1}^{+}=\cos (\theta x)-\left(\frac{\cos \left(\frac{\theta H}{2}\right)}{\cosh \left(\frac{\omega H}{2}\right)}\right) \cosh (\omega x) \\
& \Phi_{s 2}=\frac{k}{v \Sigma_{f 2}}\left(\left(D_{1} \mu^{2}+\Sigma_{R 1}\right) \cos (\mu x)-\left(\Sigma_{R 1}-D_{1} \lambda^{2}\right)\left(\frac{\cos \left(\frac{H}{2} \mu\right)}{\cosh \left(\frac{H}{2} \lambda\right)}\right) \cosh (\lambda x)\right), \\
& \Phi_{s 2}^{+}=\frac{1}{\Sigma_{R 1}}\left(\left(D_{1} \theta^{2}+\Sigma_{R 1}\right) \cos (\theta x)-\left(\Sigma_{R 1}-D_{1} \omega^{2}\right)\left(\frac{\cos \left(\frac{\theta H}{2}\right)}{\cosh \left(\frac{\omega H}{2}\right)}\right) \cosh (\omega x)\right),
\end{aligned}
$$


onde $\mu, \lambda, \theta$ e $\omega$ são constantes definidas da seguinte maneira:

$\mu=\sqrt{\frac{\left(\left(D_{1} \Sigma_{a 2}+D_{2} \Sigma_{R 1}\right)+\sqrt{\left(D_{1} \Sigma_{a 2}+D_{2} \Sigma_{R 1}\right)^{2}+4 D_{1} D_{2} \Sigma_{R 1}\left(\Sigma_{a 2}-\frac{v \Sigma_{f 2}}{k}\right)}\right.}{2 D_{1} D_{2}}}$,

$\lambda=\sqrt{\frac{-\left(D_{1} \Sigma_{a 2}+D_{2} \Sigma_{R 1}\right)+\sqrt{\left(D_{1} \Sigma_{a 2}+D_{2} \Sigma_{R 1}\right)^{2}+4 D_{1} D_{2} \Sigma_{R 1}\left(\Sigma_{a 2}-\frac{v \Sigma_{f 2}}{k}\right)}}{2 D_{1} D_{2}}}$,

$\theta=\sqrt{\frac{-\left(\frac{\Sigma_{R 1}}{D_{1} D_{2}}\left(\frac{D_{1} \Sigma_{a 2}}{\Sigma_{R 1}}+D_{2}\right)+\sqrt{\left(\frac{\Sigma_{R 1}}{D_{1} D_{2}}\left(\frac{D_{l} \Sigma_{a 2}}{\Sigma_{R 1}}+D_{2}\right)\right)^{2}+4\left(\frac{v \Sigma_{f 2}}{k}-\Sigma_{a 2}\right)}\right)}{2}}$,

e

$\omega=\sqrt{\frac{\left(\frac{\Sigma_{R 1}}{D_{1} D_{2}}\left(\frac{D_{1} \Sigma_{a 2}}{\Sigma_{R 1}}+D_{2}\right)+\sqrt{\left(\frac{\Sigma_{R 1}}{D_{1} D_{2}}\left(\frac{D_{1} \Sigma_{a 2}}{\Sigma_{R 1}}+D_{2}\right)\right)^{2}+4\left(\frac{v \Sigma_{f 2}}{k}-\Sigma_{a 2}\right)}\right)}{2} .}$

Novamente, foram obtidos os valores numéricos dos parâmetros cinéticos para o sistema crítico e o sistema subcrítico acionado por fonte, utilizando as expressões dos fluxos e as Equaçoes (2.17) e (2.21). O comportamento dos parâmetros em função do fator de multiplicação, $K_{e f f}$, é visualizado nas Figuras 7,8 e 9.

Note que no caso de 2 grupos, o fator de multiplicação é obtido através da condição de que a equação homogênea da (2.44) tivesse não apenas soluções triviais [40]:

$$
K_{e f f}=\frac{v_{1} \Sigma_{f 1}}{\Sigma_{R I}+D_{I} B_{g}^{2}}+\frac{\Sigma_{R 1}}{\left(\Sigma_{R I}+D_{I} B_{g}^{2}\right)} \frac{v_{2} \Sigma_{f 2}}{\left(\Sigma_{a 2}+D_{2} B_{g}^{2}\right)} .
$$


Vale lembrar que neste trabalho foi assumido que a $\Sigma_{f l}$ fosse nula, ou seja, a fissão ocorre somente no grupo térmico.

Como no caso anterior, a largura crítica do reator foi calculada impondo a condição da criticalidade, isto é: $K_{\text {eff }}=1$, e a largura do sistema subcrítico foi calculada para $K_{\text {eff }}=0,98$. Os dados nucleares utilizados no cálculo são do Reator IPEN-MB-01 [45], e se encontram na Tabela 3. As larguras crítica e subcrítica calculadas são 28,65777176 cm e 27,85921574 cm, respectivamente.

\begin{tabular}{|c|c|c|c|c|}
\hline Grupo & $\boldsymbol{D}(\mathrm{cm})$ & $\Sigma_{a}\left(\mathrm{~cm}^{-1}\right)$ & $\boldsymbol{v} \Sigma_{f}\left(\mathrm{~cm}^{-1}\right)$ & $\Sigma_{\boldsymbol{R}}\left(\mathrm{cm}^{-1}\right)$ \\
\hline 1 & 1,10162 & $9,06423 \mathrm{e}-3$ & $7,33979 \mathrm{e}-3$ & $2,71802 \mathrm{e}-2$ \\
\hline 2 & 0,239190 & $1,22190 \mathrm{e}-1$ & $1,85980 \mathrm{e}-1$ & $3,75949 \mathrm{e}-2$ \\
\hline
\end{tabular}

Tabela 3. Dados do sistema para 2 grupos de energia [45]

A a velocidade média do grupo rápido do problema foi calculada assumindo que a energia média do grupo fosse $1,45 \mathrm{MeV}$ [25]. Para o grupo térmico foi assumida que a velocidade média fosse $2,2 \times 10^{5} \mathrm{~cm} / \mathrm{s}$ [44].

Os valores obtidos encontram-se na Tabela A.1.2 no Apêndice, e os gráficos são apresentados nas Figuras 7, 8 e 9. Os comportamentos dos parâmetros cinéticos mostrados nesses gráficos são análogos aos dos obtidos no modelo de 1 grupo de energia, embora os dois problemas se tratem de sistemas de composição e geometria diferentes. Observe que os gráficos dos parâmetros cinéticos dos ambos modelos estão em escalas diferentes. Nesses gráficos observamos novamente que os $\beta$ e $\Lambda$ clássicos se mantem praticamente constantes em relação à variação do $K_{\text {eff, }}$, enquanto os $\beta_{s} \mathrm{e}$ $\Lambda_{s}$ variam linearmente com $K_{\text {eff }}$ e tendem aos $\beta$ e $\Lambda$, respectivamente, quando $K_{\text {eff }}$ tende a 1 . Quanto aos parâmetros $\rho$ e $\rho_{s, o}$, ambos variam com o grau da subcriticalidade do sistema, mas a diferença entre os ambos diminui com $K_{\text {eff }}$ tendendo a 1.

Nota-se que $\rho_{s}$ é a grandeza que de certa forma expressa a "reatividade generalizada", e de alguma maneira é relacionada com a variação percentual de $k_{s}$, isto é, $\delta k_{s} / k_{s}$, enquanto $\rho_{s, 0}$ expressa somente a presença da fonte $\left(\rho_{s, 0}\right.$ está contido em $\left.\rho_{s}\right)$. 


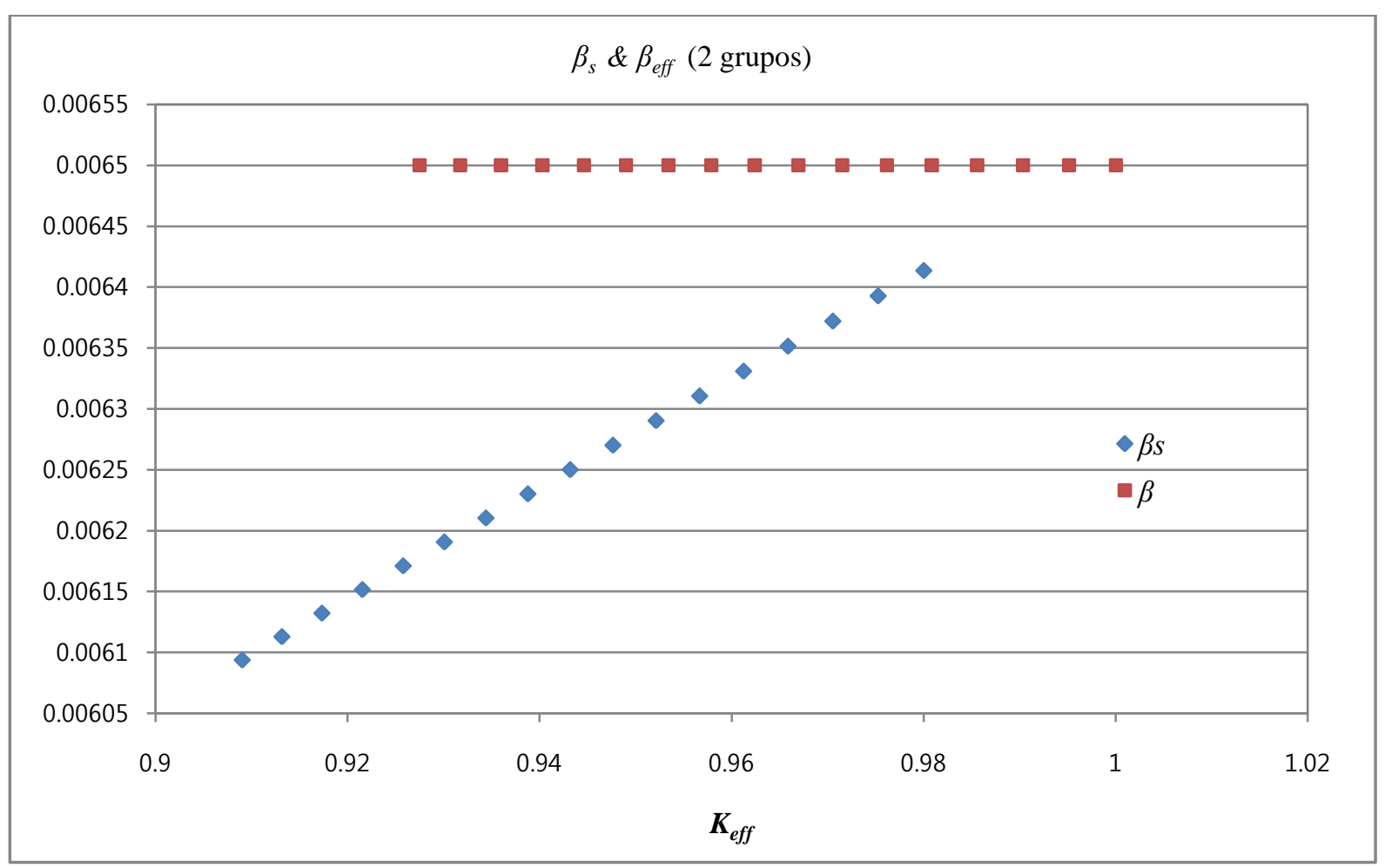

Figura 7. Os valores dos $\beta_{\text {eff }}$ e $\beta_{s}$ em função do $K_{\text {eff }}$ no modelo de 2 grupos.

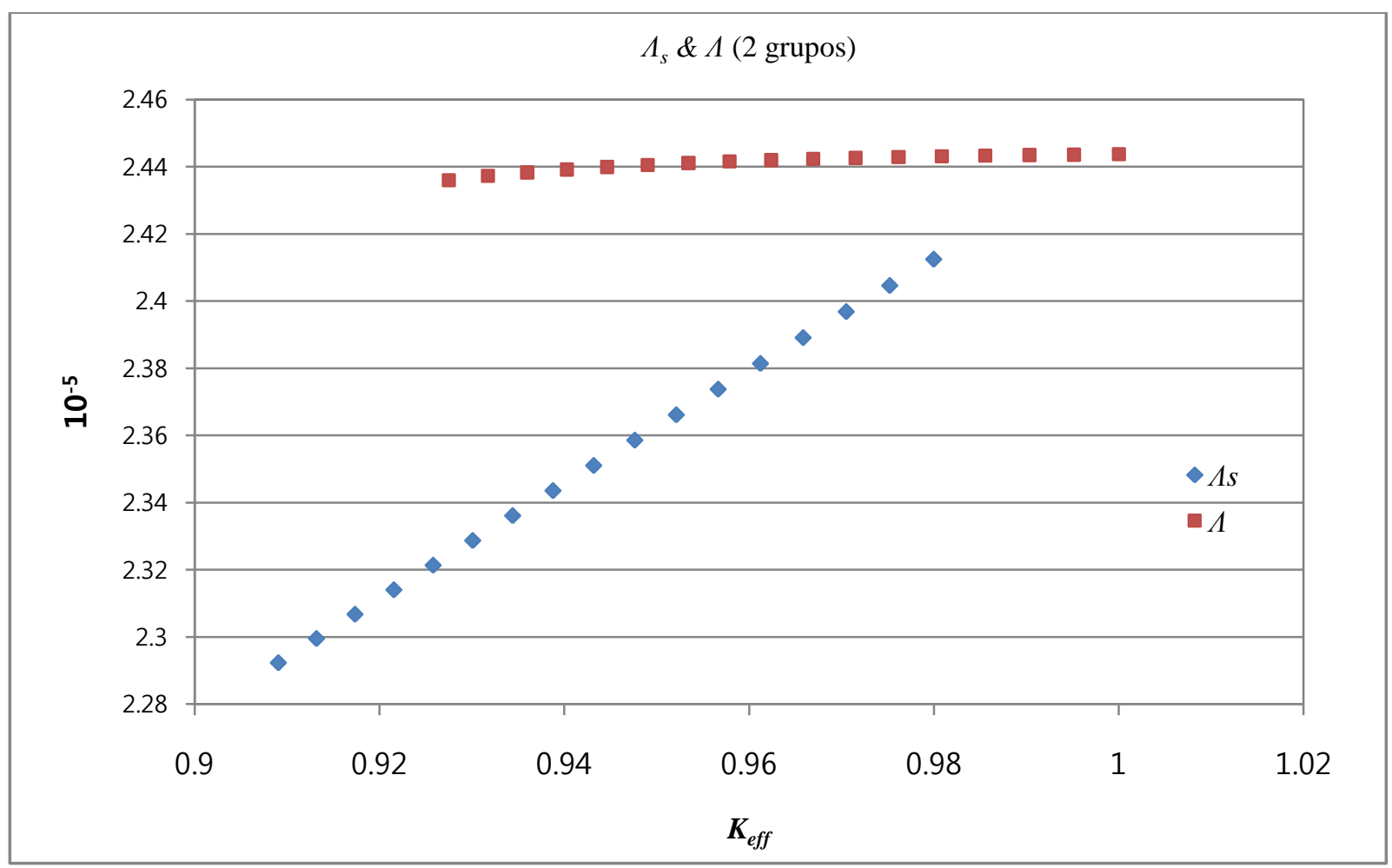

Figura 8. Os valores dos $\Lambda$ e $\Lambda_{s}$ em função do $K_{\text {eff }}$ no modelo de 1 grupo. 


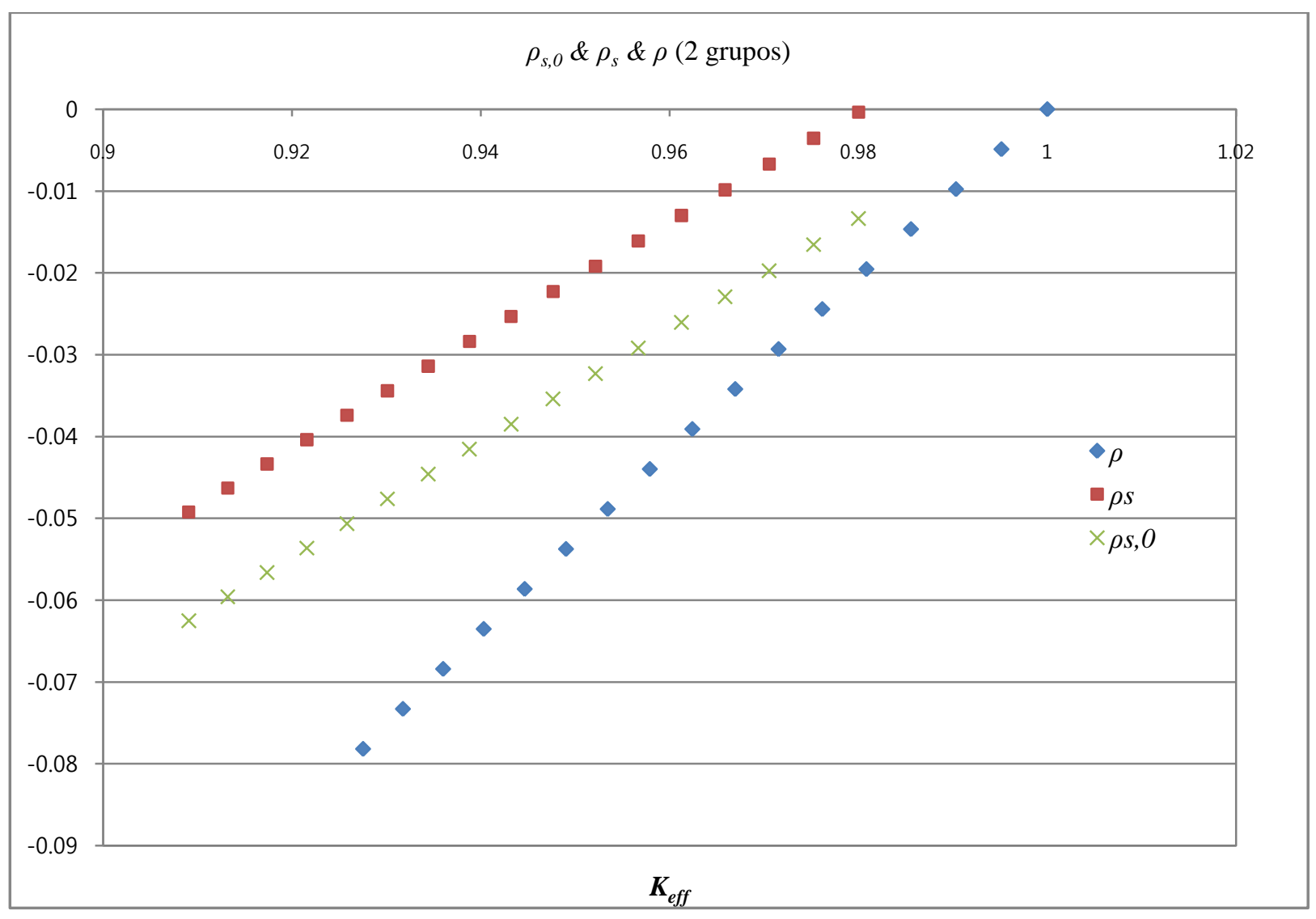

Figura 9. Os valores de $\rho, \rho_{s, 0}$ e $\rho_{s}$ em função do $K_{\text {eff }}$ no modelo de 2 grupos. 


\section{MÉTODOS EXPERIMENTAIS DE MEDIADAS DE PARÂMETROS CINÉTICOS}

As técnicas de medida dos parâmetros cinéticos em sistemas críticos, também denominadas "in pile" datam dos primórdios da Física dos Reatores na década de 50 e 60, e assumem na maioria dos métodos a validade da cinética pontual, ou correções espaciais destas. Basicamente estas técnicas são: o método de queda de barras ou "rod drop", pequenas inserções de reatividade, oscilação de reatividade, e mais recentemente o método de transientes múltiplos, desenvolvido por Spring na década de 90 [46], e aplicado no Reator Brasileiro IPEN-MB-01 por Santos et al. [47], e finalmente a técnica de análise de Ruído, também aplicada no IPEN-MB-01 por Diniz et al. [48], além das técnicas de fontes pulsadas. Neste trabalho, pretende-se rever estas técnicas, tendo em vista principalmente uma avaliação crítica destas com relação aos modelos adotados (cinética pontual), e consolidar o "estado da arte" nestas técnicas de medida.

Por outro lado, em sistemas subcríticos acionados por fontes, as técnicas de medida são baseadas em medidas "out of pile", e várias destas foram também desenvolvidas nas décadas de 50 e 60. Recentemente, com o interesse nos ADS, várias destas técnicas têm sido aplicadas nas instalações de potência zero, com destaque das realizadas no experimento MUSE [9,10], sendo que na Tabela 1, foram reduzidas as principais técnicas em experimentos com fontes fixas e pulsadas.

Em resumo, estas técnicas são baseadas numa variação do grau de subcriticalidade, e medidas da resposta de um detector, a estas mudanças, ou na introdução de um pulso na fonte e medidas da resposta do detector a este transiente, conforme ilustrado na Figura 10. Em geral estas técnicas medem valores relativos dos parâmetros cinéticos, tais com $\rho_{\$}=\rho / \beta$, ou $\beta / \Lambda$, além de muitas vezes serem baseadas em modelos desenvolvidos para sistemas críticos, que conforme discutidos anteriormente não são consistentes para sistemas subcríticos acionados por fonte. 
Por outro lado, em tese de doutoramento no IPEN, Kuramoto et al. [15] introduziu um novo método de medida absoluto destes parâmetros, baseado na técnica Rossi- $\alpha$ e um modelo de múltiplos pontos. Neste trabalho, pretende-se também avaliar o "estado da arte" destas técnicas de medida, com uma visão crítica dos modelos teóricos utilizados para embasamento destas técnicas, principalmente tendo em vista o apresentado anteriormente.

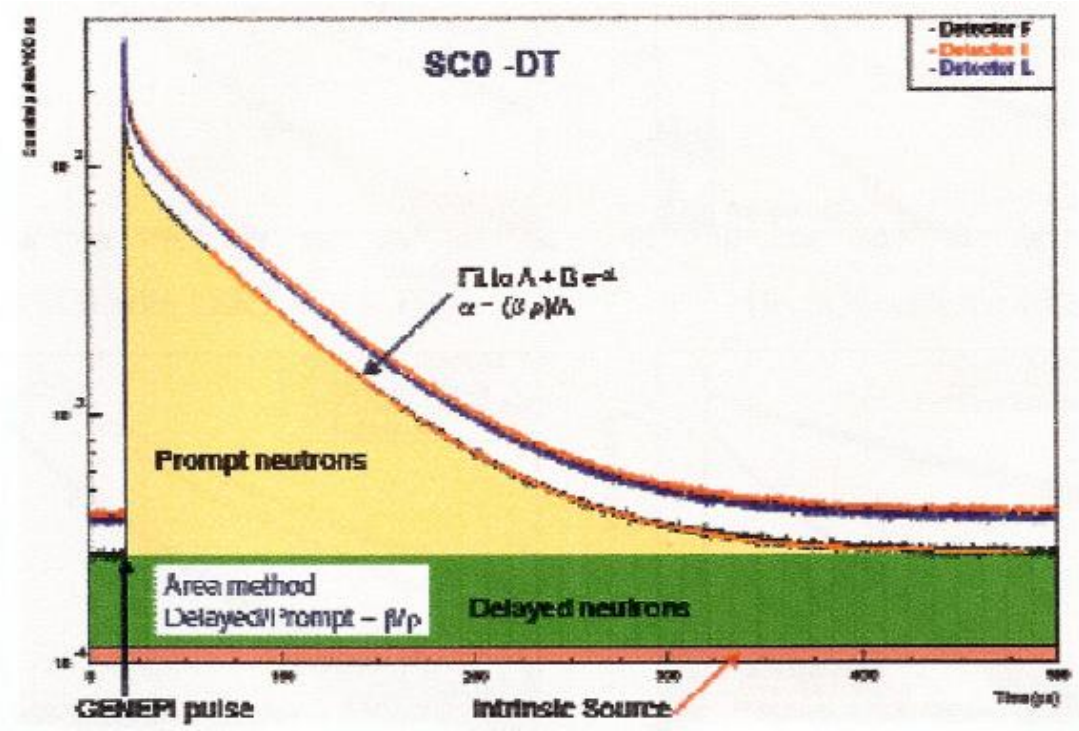

Figura 10. Ilustração esquemática típica da resposta de um detector à introdução de um pulso, para medida de parâmetros cinéticos.

\subsection{Método da fonte de ${ }^{252} \mathrm{Cf}[49]$}

Este método foi aplicado para medidas de $\beta_{\text {eff }}$ em vários sistemas críticos por: Carpenter [50], em 1972; Fisher [51], em 1977; Avramov et al. [52], em 1990; e Bertrand [53], em 1996. Neste método, $\beta_{\text {eff }}$ é determinado pela reatividade aparente de uma fonte calibrada de ${ }^{252} \mathrm{Cf}$.

O parâmetro $\beta_{\text {eff }}$ é determinada pela seguinte fórmula [51]: 


$$
\beta_{e f f}=\frac{S_{C f}}{\bar{v} \rho_{C f} F_{c} F_{r}}\left(\frac{\Phi_{C f}^{+}}{\Phi_{f u e l}^{+}}\right),
$$

onde

$S_{C f} \quad$ : taxa de emissão de nêutrons da fonte calibrada de ${ }^{252} \mathrm{Cf}\left(\mathrm{s}^{-1}\right)$;

$\rho_{C f} \quad$ : reatividade aparente da fonte de ${ }^{252} \mathrm{Cf}$ no centro do núcleo (dólar);

$F_{c} \quad:$ taxa de fissão no centro do núcleo por unidade de volume $\left(\mathrm{s}^{-1} \mathrm{~cm}^{-3}\right)$;

$\bar{v} \quad$ : número médio de nêutrons emitidos por fissão no centro do núcleo;

$\Phi_{\mathrm{Cf}}{ }^{+} / \Phi_{\text {fuel }}{ }^{+}$: razão entre as importância dos nêutrons no centro do núcleo; e

$F_{r} \quad$ : integral de normalização dado por:

$$
F_{r}=\frac{\int_{\text {reactor }}\left(\int \chi \Phi d E\right)\left(\int v \Sigma_{f} \Phi d E\right) d \mathrm{~V}}{\left(\int \chi \Phi d E\right)_{0}\left(\int v \Sigma_{f} \Phi d E\right)_{0}},
$$

onde

$\chi \quad$ : espectro da fissão dependente da região;

$\Phi, \Phi^{+}$: fluxo e fluxo adjunto dependentes da posição e energia;

$v \quad$ : número de nêutrons emitidos por fissão dependente da região e energia; e

$\Sigma_{f} \quad$ : seção de choque macroscópica de fissão dependente da região e energia.

Note que, o índice 0 nas integrais indicam que as mesmas são calculadas no centro do núcleo.

Os parâmetros $S_{C f}, \rho_{C f}$ e $F_{c}$ são medidos; o valor semi-empírico da $F_{r}$ pode ser obtida pela combinação de medidas de taxa de fissão, reatividade da fonte e fatores de correção calculados. Os $\bar{v}$ e $\Phi_{\mathrm{Cf}}^{+} / \Phi_{\text {fuel }}^{+}$são calculados.

A $\rho_{C f}$ é inversamente proporcional ao nível do fluxo, ao passo que, a $F_{c}$ é diretamente proporcional ao fluxo. Para remover a dependência em fluxo, $\rho_{C f}$ é como o produto $\rho_{C f} \cdot m$, onde $m$ é taxa de contagem de um monitor de fluxo, e $F_{c}$ como a razão $F_{c} /$ $m$. O produto desses dois parâmetros é usado para determinar $\beta_{\text {eff. }}$. 


\subsection{Método de Densidades Espectrais [54]}

Nos experimentos envolvendo medidas de densidades espectrais CPSD ("Cross Power Spectral Density"), também chamados de análise de ruído macroscópico, $\beta$ eff é determinado da seguinte maneira:

$$
\beta_{e f f}^{2}=\frac{2 D V_{1} V_{2}}{F_{c} F_{r} C P S D(1+|\rho|)^{2}},
$$

onde

$D \quad$ : fator de dispersão ou de Diven, definido como[55]:

$$
\begin{gathered}
D=\frac{\overline{v_{p}\left(v_{p}-1\right)}}{v_{p}^{2}}, \\
\text { onde } v_{p}=v(1-\beta) ;
\end{gathered}
$$

$V_{l, 2} \quad$ : quantidades proporcionais às correntes nas câmaras de ionização 1 e 2 utilizadas para normalizar a CPSD; e

$\rho \quad: \quad$ valor absoluto da reatividade do núcleo.

Os demais parâmetros possuem as mesmas definições mencionadas previamente.

Os parâmetros $D$ e $F_{r}$ são obtidos semi-empiricamente. Com exceção da reatividade, os demais parâmetros são obtidos diretamente das densidades espectrais.

\subsection{Método Rossi- $\alpha$}

O método Rossi- $\alpha$ [49], sugerido por Bruno Rossi [56, 57, 58], foi desenvolvido 
para reatores rápidos onde o tempo de vida do nêutron é extremamente curto. Posteriormente, Feynman, Hoffman e Serber [59] desenvolveram um tratamento estatístico das cadeias de fisssão. Mais tarde, Matthes [60], Borgwaldt [61], Babala [62], e Iijima [63] desenvolveram um tratamento com um maior rigor matemático.

No método Rossi- $\alpha$ tradicional, $\beta_{\text {eff }}$ é obtido da razão entre a parte correlacionada da distribuição Rossi- $\alpha$ e do fundo através da relação:

$$
\beta_{\text {eff }}=\frac{1}{1+\left(1-\rho_{\$}\right) \sqrt{\frac{2 F_{c} F_{r}}{D}\left(\frac{C_{\text {corr }}}{C_{\text {rand }}}\right) \Delta t \frac{(\alpha+C)}{\alpha}}},
$$

onde

$C_{c o r r} \quad$ : número total de contagens na parte correlacionada da curva Rossi- $\alpha$;

$C_{\text {rand }}:$ número médio de contagens por canal na região do fundo da curva Rossi- $\alpha$;

$\Delta t \quad$ : largura do canal;

$C \quad$ : taxa de contagem no detector que adquire a distribuição Rossi- $\alpha$;

$\alpha \quad$ : constante de decaimento de nêutron pronto; e

os demais parâmetros tem definições idênticas das seções anteriores.

Com excepção de $D$ e $F_{r}$, que são semi-empíricos, todo os parâmetros são experimentais, sendo $C_{c o r r}, C_{\text {rand }}, \Delta t, C$ e $\alpha$, obtidos diretamente dos experimentos Rossi- $\alpha$.

\subsection{Método do Número de Nelson}

No método do número de Nelson, uma fonte externa com intensidade conhecida, é inserida no sistema multiplicativo para dar início à fonte intrínseca estacionária, e assim, começar cadeias de fissão. Neste método, os dados fundamentais são obtidos de distribuições Rossi- $\alpha$. Medindo a área sob a curva Rossi- $\alpha$ adquirida, $\beta_{\text {eff }}$ é determinado por intermédio da 
relação [64]:

$$
\beta_{e f f}=-\frac{\rho_{\$}}{N\left(1-\rho_{\$}\right)^{2}-\rho_{\$}},
$$

onde $N$ é o número de Nelson definida como[64]:

$$
\Delta=\left(\frac{2 g^{*} S}{g \bar{v}_{p} D}\right)\left(\frac{A}{\alpha C}\right),
$$

onde

$S \quad$ : intensidade total da fonte de nêutrons (a inicial e a intrínseca)

$g$ e $g^{*}$ : fatores de correção espacial [65];

$\bar{v}_{p} \quad$ : número de nêutrons prontos emitidos por fissão;

A : valor da distribuição da curva na interseção com a ordenada;

$\alpha \quad$ : inclinação da curva da distribuição; e

$C \quad$ : taxa de contagem no detector que mediu a curva de Rossi- $\alpha$.

A quantidade $g * S$ é equivalente à intensidade da fonte no modo fundamental [66], e $g D$ o fator de Diven com ponderação adjunta.

A reatividade é determinada usando a relação:

$$
\rho=1-\frac{\alpha^{*}}{\alpha_{0}},
$$

onde $\alpha_{0}$ é constante de decaimento correspondente a crítico atrasado; e $\alpha^{*}$ é $\alpha$ ajustado da seguinte maneira:

$$
\alpha^{*}=\alpha \sqrt{\left(\frac{(A \alpha)_{0}}{A \alpha}\right)} \text {. }
$$

Novamente, o parâmetro $D$ é semi-empírico. Os demais parâmetros são obtidos 
através de análises das curvas de Rossi- $\alpha$.

\section{Método Feynman- $\alpha$ [49]}

Este método é baseado na medida da razão entre a variância e a média do número de contagens adquiridas em um intervalo de tempo fixo. Para reatores térmicos onde o tempo de vida dos nêutrons é longo, o método Feynmann- $\alpha$ é mais adequado que o método Rossi- $\alpha$.

Foi mostrado por Feynmann et al. [59], que o número de contagens $c$ em um intervalo de tempo $T$, desvia de uma distribuição de Poisson devido à flutuações na população neutrônica. Esse desvio é medido pela quantidade $Y$ que corresponde à razão entre a variância $\sigma^{2}$ e o valor médio das contagens $c$. Ou seja:

$$
Y(T)=\frac{\sigma^{2}}{\bar{c}}-1=\frac{\left(\overline{c^{2}}-\overline{c^{2}}\right)}{\bar{c}}-1 .
$$

A distribuição $Y(T)$ é obtida experimentalmente através de uma técnica chamada técnica de "bunching", proposta por Misawa et al. [67, 68].

Neste método, $\beta_{\text {eff }}$ é obtido da seguinte fórmula [49]:

$$
\beta_{\text {eff }}=\frac{1}{1+\left(1-\rho_{\$}\right) \sqrt{\frac{a_{p} F_{c} F_{r}}{\epsilon D}}},
$$

onde

$$
\begin{array}{ll}
a_{p} & : \text { a amplitude a distribuição de Feynmann- } \alpha ; \mathrm{e} \\
\varepsilon & : \text { a eficiência do detector. }
\end{array}
$$




\subsection{Método de Bennett ou da Covariância [69]}

Este método foi proposto por Bennett[70] em 1981. Uma das primeiras medidas de $\beta_{\text {eff }}$ de um sistema crítico (rápido) foi efetuada em KUCA (Kyoto University Critical Assembly) em 1992 [71]. Neste método é medida a covariância entre as contagens registradas por dois detectores de nêutrons.

A fórmula original para $\beta_{\text {eff }}$ é:

$$
\beta_{\text {eff }}=\frac{1}{1+\left(1-\rho_{\$}\right) \sqrt{\frac{F_{c} F_{r}}{D} A_{0}}},
$$

onde $A_{0}$ é obtida ajustando a amplitude da curva da covariância em função da largura em tempo do canal. Novamente, $D$ e $F_{r}$ são obtidas semi-empiricamente, enquanto $F_{c}$ e $\rho_{\$}$ são obtidos experimentalmente.

Em uma versão modificada o método de Bennett, a expressão para o $\beta_{\text {eff }}$ tem um termo a mais, $D_{s}$, na frente do fator de Diven, $D$, da fórmula original. Essse termo é o fator de correção espacial sugerido por Otsuka e Iijima [72].

Todos os métodos apresentados até aqui medem o $\beta_{\text {eff }}$ e, excepto o Método de Densidedes Espectrais, usam o termo $\rho_{\$}$, que é definido como a razão entre a reatividade $\rho$ e $\beta_{\text {eff. }}$ Ou seja, para determinar o $\beta_{\text {eff }}$ precisa saber do próprio $\beta_{\text {eff }}$ ! Como se determina previamente o $\rho_{\$}$ ? Será por algum método de fonte de nêutrons pulsada que serão discutidos a seguir?

\subsection{Métodos de Fonte de Nêutrons Pulsada}

Quando um pulso de nêutrons entra em um núcleo de um sistema subcrítico, começa 
a propagar-se um certo número de cadeia de fissão dentro do combustível. Porém, toda cadeia de fissão é rapidamente cessada por causa da subcriticalidade do sistema. Esse fenômeno é caracterizado por um decaimento global do fluxo de nêutrons de forma exponencial. Desta forma é possível determinar a reatividade do núcleo através de uma análise desse decaimento de nêutrons prontos. Existem dois métodos de análise:

\subsubsection{Método de "Slope Fit" ou Método de Ajuste de Inclinação}

Este método foi introduzido pela primeira vez por Simmons [73], em 1958. Mas somente nos últimos anos começou a chamar a atenção devido a sua possível aplicabilidade nos ADS.

Desprezando o efeito dos nêutrons atrasados e a fonte externa, a equação da cinética pontual, em termos de $n(t)$, densidade de número de nêutrons, pode ser escrita da seguinte forma [39]:

$$
\frac{d n(t)}{d t}=\alpha n(t)
$$

cuja solução é:

$$
n(t)=n_{0} e^{\alpha t}
$$

onde $\alpha$ é a constante de decaimento de nêutrons prontos:

$$
\alpha=\frac{\left(\rho-\beta_{e f f}\right)}{\Lambda}
$$

Para um sistema subcrítico a constante $\alpha$ é negativa, ou seja, o fluxo de nêutrons decresce exponencialmente; e num pequeno intervalo de tempo após a inserção do pulso, da ordem de milisegundos, o efeito dos nêutrons atrasados pode ser desprezado; desta forma, o decaimento é essencialmente dos nêutrons prontos. A constante $\alpha$ é medida 
experimentalmente, e a reatividade é obtida uma vez que $\beta_{\text {eff }}$ e $\Lambda$ são conhecidos. A Figura 11 [39] mostra um ajuste exponencial aos dados experimentais.

Aproximadamente $4 \mathrm{~ms}$ depois, o fluxo de nêutrons atinge o modo fundamental de decaimento; este fenômeno é descrito por equações de cinética pontual. Durante este processo, todos os canais registram quase a mesma taxa de decaimento. E, finalmente, o fluxo atinge o nível constante devido ao fundo dos nêutrons atrasados.

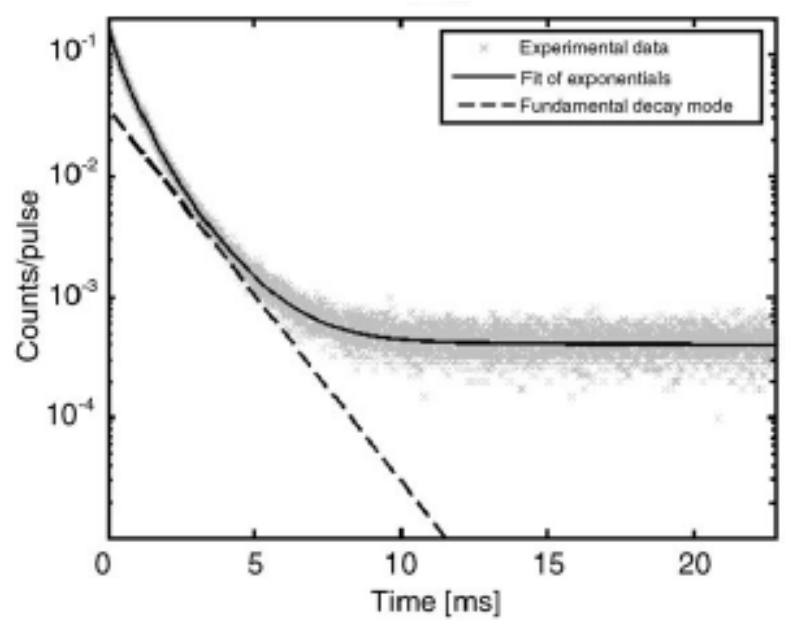

Figura 11. Ajuste exponencial aos dados experimentais de um canal no interior do núcleo [39].

De acordo a este método, a constante $\alpha$ é medida experimentalmente e, se $\Lambda$ for conhecido pode se dizer que foi medida a reatividade em dólar, $\rho_{\S}\left(\rho_{\$} \equiv \rho / \beta_{e f f}\right)$. Mas, como será que se obtém o $\Lambda$ ?

Por outro lado, a constante de decaimento $\alpha$ não dependeria do tipo, da intensidade e do período do pulso? Por exemplo, a $\alpha$ obtida usando um pulso quadrado de intensidade $I_{1}$ com um período $T=\Delta t_{1}$ seria a mesma da obtida usando um outro pulso triangular de intesidade $I_{2}$ de base $\Delta t_{2}$ ? Se os dois resultados fossem diferentes, não seriam obtidas duas $\rho_{\S}$ diferentes que, por definição, são próprias de cada sistema? Acaso estamos considerando dois sistemas diferentes? 


\subsubsection{Método de Sjöstrand ou Método das Áreas}

Considerando a escala de tempo de decaimento do fluxo de nêutrons prontos muito menor em relação à meia-vida dos precursores de nêutrons atrasados, a contribuição dos nêutrons atrasados pode ser considerada constante durante o intervalo de tempo. Se a área sob a curva da função resposta for dividida em duas partes, uma área de nêutrons prontos, $A_{p}$, e outra de nêutrons atrasados, $A_{d}$, como é mostrada na Figura 12, a reatividade (em dólar) pode ser expressada como [74]:

$$
\frac{\rho}{\beta_{\text {eff }}}=-\frac{A_{p}}{A_{d}} .
$$

A área de nêutrons prontos é obtida através da integral numérica trapezoidal, e a $A_{d}$ pelo valor médio nos últimos milisegundos, onde a curva torna-se horizontal.

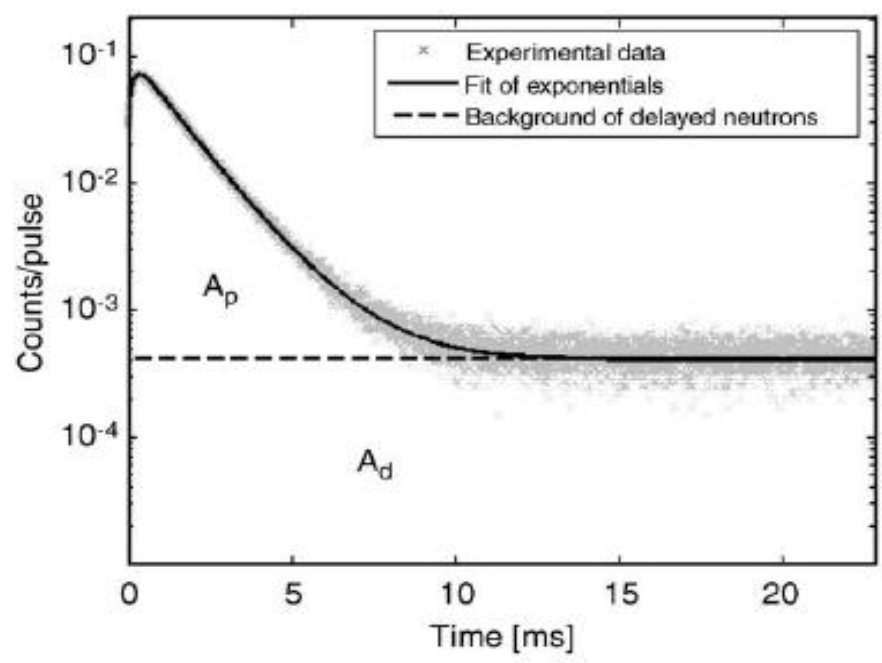

Figura 12. Ilustração das áreas de nêutrons prontos e de nêutrons atrasados utilizados no Método de Sjöstrand[39].

\subsection{Experiência de "Source Jerk"}

Se um reator subcrítico operando inicialmente no estado estacionário, em um nível de 
fluxo, $n_{0}$, sofrer súbita remoção da fonte, será observado um queda do sistema ou "prompt jump" para um nível inferior, $n_{l}$, determinado pelo fundo ou "background" de nêutrons atrasados. Essse nível é simplesmente quase-estático e vai decair de acordo com a taxa de decaimento dos precursores de nêutrons atrasados. A reatividade em dólar é dada por [25]:

$$
\frac{\rho}{\beta_{e f f}}=\frac{n_{1}-n_{0}}{n_{1}}
$$

A Figura 13 mostra o fluxo de nêutrons medido por um detector de ${ }^{3} \mathrm{He}$ em uma experiência realizada em YALINA. Os níveis de fluxos são estimados antes e logo depois da queda; desta forma obtém-se a reatividade. Devido à baixa contagem após a queda, o erro estatístico é considerável.

\subsection{Método da Reatividade Inversa}

A reatividade pode ser calculada a partir das equações da cinética pontual isolando o termo da reatividade. Das Equações (2.16) obtida pelo método clássico, obtém-se a seguinte expressão da reatividade:

$$
\rho(t)=\beta_{e f f}+\Lambda \frac{d[\ln P(t)]}{d t}-\beta_{e f f} \int_{0}^{\infty} D(\tau) \frac{P(t-\tau)}{P(t)} d \tau
$$

onde

$$
D(\tau)=\sum_{i=1}^{6} \frac{\lambda_{i} \beta_{i}}{\beta} e^{-\lambda_{i} \tau}
$$

Porém, a partir das Equações (2.20) obtidas para sistemas subcríticos acionados por fonte utilizando as novas definições dos parâmetros, um procedimento análogo para obter a expressão anterior gera uma expressão diferente para a reatividade, $\rho_{s}$ : 


$$
\rho_{s}=\beta_{s}+\frac{\Lambda_{s}}{P(t)}\left[\frac{d P(t)}{d t}-\lambda \beta_{s} \int_{0}^{t} P\left(t^{\prime}\right) e^{-\lambda\left(t-t^{\prime}\right)} d t^{\prime}-\Lambda_{s} \mathbb{S}\right] .
$$

Como foi mostrado no Capítulo 2, tanto $\rho_{s}$, como $\rho$ dependem do grau da subcriticalidade, mas $\rho_{s}$ depende de perturbação, também. Conseqüentemente surge uma pergunta: "Para que serve um parâmetro que depende tanto do grau da subcriticalidade como da perturbação?”.

\subsection{Modelo de Duas Regiões}

A Cinética Pontual para duas regiões é um modelo desenvolvido para descrever satisfatoriamente os resultados experimentais obtidos em reatores refletidos, que são compostos de duas regiões: o núcleo e o refletor.

Um método para determinação absoluta de $\beta_{\text {eff }}$ fundamentada no Modelo de Duas Regiões foi desenvolvido no Reator IPEN/MB-01 [47,49]. Este método está baseado no comportamento não-linear entre a constante de decaimento de nêutrons prontos, $\alpha$, e a reatividade $\rho$. Segundo o Modelo de Duas Regiões este comportamento é descrito através da solução da equação inhour para o caso em que se considera um intervalo de tempo muito pequeno comparado com as meia-vidas dos precursores:

$$
\begin{aligned}
& \omega_{7,8}=\frac{1}{2 \tau_{c} \tau_{r}(1-\rho)}\left\{\left[(1-\rho)\left(f \tau_{c}+\tau_{r}\right)+\tau_{r}\left(\beta_{\text {eff }}-\rho\right)(1-f)\right] \pm\right. \\
& \left. \pm\left(\left[(1-\rho)\left(f \tau_{c}+\tau_{r}\right)+\tau_{r}\left(\beta_{e f f}-\rho\right)(1-f)\right]^{2}-4 \tau_{c} \tau_{r}(1-f)(1-\rho)\left(\beta_{e f f}-\rho\right)\right)^{\frac{1}{2}}\right\},
\end{aligned}
$$

onde

$$
\begin{array}{lll}
\omega_{7,8} & : & \text { raízes da equação inhour } \omega_{7} \text { e } \omega_{8} ; \\
\tau_{c} & : & \text { tempo de vida ponderado dos nêutrons no núcleo; } \\
\tau_{r} & : & \text { tempo de vida ponderado dos nêutrons no refletor; }
\end{array}
$$


$f \quad$ : $\quad$ fração de retorno do refletor.

Ajustando a curva de $\alpha$ versus $\rho$ com a Equação (3.17) é possível determinar os $\beta_{\text {eff, }}$ $\tau_{c}, \tau_{r}$ e $f$. Para poder observar o comportamento não-linear é necessário adquirir os dados em um grande intervalo de subcriticalidade.

$\mathrm{O} \Lambda$ é calculado usando a relação direta com os parâmetros $\tau_{c}, \tau_{r}$ e $f$ determinados acima:

$$
\Lambda=\frac{1}{1-f}\left(\tau_{c}+f \tau_{r}\right)
$$

onde foi assumido que o tempo de vida dos nêutrons no refletor seja suficientemente pequeno, o que vale na maioria dos reatores refletidos. 


\section{CONCLUSÃO E RECOMENDAÇÕES}

Neste trabalho mostrou-se que os chamados parâmetros cinéticos necessitam de uma definição mais consistente para sistemas subcríticos acionados por fontes.

A grandeza $\rho=\left(K_{\text {eff }}-1\right) / K_{\text {eff, }}$ para sistemas subcríticos acionados por fontes não é a "reatividade", mas sim o desvio da criticalidade.

As medidas dos parâmetros cinéticos necessitam uma melhor interpretação, tendo em vista a dependência destes parâmetros com o grau da subcriticalidade e a perturbação.

Usar um reatímetro, utilizando uma cinética inversa, e colocando alguns valores de $\beta$ e $\Lambda$, obtidos para sistemas críticos, fornecerá uma informação que não indica o desvio da criticalidade, $\left(K_{\text {eff- }} 1\right) / K_{\text {eff. }}$ O que é fornecido necessita, ainda, uma melhor investigação. Podese, talvez da Equação (2.24) inferir-se sobre $\delta k_{s} / k_{s}$.

Como sugestão de trabalhos futuros, deve-se investigar principalmente a questão de reatímetro, e uma demonstração experimental.

Por outro lado, os espectros de energia de nêutrons prontos e de nêutrons atrasados são bem diferentes, mas o efeito dessa diferença nos cáculos dos parâmetros cinéticos é praticamente perdido quando se trabalha com apenas 1 ou 2 grupos de energias. No modelo de 2 grupos de energias, por exemplo, os nêutrons atrasados perdem suas identidades no vasto grupo rápido, e os efeitos causados por esses são minimizados. Portanto, como outra sugestão de trabalhos futuros propõe-se estender o análise para mais grupos de energia e famílias de precursores para poder visualizar melhor este efeito. É esperado que o parâmetro mais afetado pelo aumento de número de grupos de energia seja o $\beta_{s}$, e para complementar, sugere-se levar em consideração a realimentação termohidráulica, que foi ignorada neste trabalho, pois assumiu-se de antemão, que o sistema fosse de "potência zero".

A aplicação de uma nova cinética pontual na região subcrítica com as novas interpretações ou definições dos parâmetros cinéticos e suas medidas experimentais 
adequadas é um assunto sério e a comunidade internacional de energia nuclear deve perceber disso. Tem um refrão coreano que diz: "Quem apanha primeiro sofre menos”. Largar velhos hábitos sempre causam crises, mas se uma crise é inevitável seria melhor enfrentá-la logo. Embora não exista nenhum reator subcrítico de potência em operação, quase todo sistema crítico começa apartir do estado subcrítico; se o comportamento dinâmico não for corretamente interpretado, como poderemos garantir a análise de segurança dos reatores? 


\section{REFERÊNCIAS BIBLIOGRÁFICAS}

[1] IAEA, Review of National Accelerator Driven System Programmes for Partitioning and Transmutation, IAEA TECDOC 1365, August 2003.

[2] W. GUDOWSKI (Ed), Accelerator Driven Systems: Energy Generation and Transmutation of Nuclear Waste, Status Report, IAEA-TECDOC 985, IAEA Vienna, 1997.

[3] S. TACZANOWSKI, Transmutation of Nuclear Waste in Accelerator-Driven Subcritical Systems, Applied Energy 75, pp. 97-117, 2003.

[4] W. GUDOWSKI, Accelerator-driven Transmutation Projects. The Importance of Nuclear Physics Research for Waste Transmutation, Nucl. Phys. A 654, 436-457, 1999.

[5] J.R MAIORINO et al., The Utilization of Accelerators in Sub Critical Systems for Energy Generation and Nuclear Waste Transmutation-The World Status and a Proposal of a National R\&D Program, Brazilian Journal of Physics, 33, 2, 2003.

[6] J.R.MAIORINO, Accelerator Driven System: An Innovative Reactor to be Used as Dedicated Waste Burner and a Multipurpose Neutron Source: The Status of the Art, International Nuclear Atlantic Conference-INAC 2005, Santos-SP, Brazil, August 28 to September 2, 2005.

[7] A. ANTUNES. de. Um Estudo da Física de Sistemas Multiplicativos Acionados por Fontes e a Utilização de Códigos Determinísticos no Cálculo destes Sistemas. 2008.Dissertação (Mestrado)-Instituto de Pesquisas Energéticas e Nucleares,São Paulo

[8] P. BENOIT et al., Small-scale LBE-cooled ADS: MYRRHA - Engineering Design Description, Proceedings of the "International Workshop on P\&T and ADS Development 2003". Mol (Belgium), October 6-8, 2003, edited by W. Haeck et al., BLG - 959, ISBN 9076971072, 2003.

[9] E. GONZALES ROMERO ET AL., The MUSE4 Pulsed Neutron Source Experiment, in PHYSOR 2004, Chicago, Illinois, April 25-29, CD Room, American Nuclear Society, 2004.

[10] M.CARTA ET AL., Reactivity Assessment and Spatial Time-effects from the MUSE Kinetics Experiment, in PHYSOR 2004, Chicago, Illinois, April 25-29, CD Room, American Nuclear Society, 2004. 
[11] V.BOURNOS et al., Experimental Investigation on ADS at Sub-Critical Facility of Joint Institute for Power and Nuclear Research-SOSNY of the National Academy of Science of Belarus, Coordinated Research Meeting on the CRP Analytical and Experimental Benchmark Analysis of ADS, Minsk, 5-9 December, 2005.

[12] C.H.PYEON et al., Present State of Accelerator Driven Subcritical Reactor (ADSR) Project in Kyoto University Research Reactor Institute (KURRI), in PHYSOR-2006 Vancouver, BC, Canada - Advances in Nuclear Analyses and Simulation, on CD Room, American Nuclear Society, ISBN 0-89448-697-7, 2006.

[13] H. AÏT-ABDERRAHIM \& A. STANCULESCU, IAEA Coordinate Research Project on "Analytical and Experimental Benchmark Analyses of Accelerator Driven Systems" in PHYSOR-2006 Vancouver, BC, Canada - Advances in Nuclear Analyses and Simulation, on CD Room, American Nuclear Society, ISBN 0-89448-697-7, 2006.

[14] J. R. MAIORINO et al., The Utilization of a Compact Neutron Generator To Drive a Sub Critical Core For Reactor Physics Experiments, Int. Nucleus-Nucleus Conf. August 28-September 1, 2006, Rio, Brazil.

[15] R. KURAMOTO et al., Rossi- $\alpha$ Experiment in the IPEN/MB-01 Research Reactor: Validation of Two-Region Model and Absolute Measurement of $\beta_{\text {eff }}$ and $\Lambda$, PHYSOR2006, ANS Topical Meeting on Reactor Physics Organized and hosted by the Canadian Nuclear Society. Vancouver, BC, Canada. 2006 September 10-14.

[16] S. DULLA et al., Kinetic Parameters for Source Driven Systems, PHYSOR-2006, ANS Topical Meeting on Reactor Physics Organized and hosted by the Canadian Nuclear Society. Vancouver, BC, Canada. 2006 September 10-14.

[17] S. DULLA et al., Integral Parameters in Source-Driven Systems: A Critical Evaluation. Second Research Coordination Meeting (RCM) of the Coordinated Research Project (CRP) on "Analytical and Experimental Benchmark Analyses of Accelerator Driven Systems (ADS)" and Technical Meeting (TM) on "Collaborative Work on LEU Fuel Utilization in ADS Systems" Ente per le Nuove Tecnologie, l'Energia e l'Ambiente (ENEA) Rome, Italy, 12 -16 November 2007.

[18] J. A. FAVORITE, W. M, STACEY, JR., "A new variational functional for spacetime neutronics", Nuclear Science and Engineering, 125, 101-106 (1997).

[19] A. FAVORITE, W. M, STACEY, JR., "Variational estimates for use with the improved quasitatics method for reactor dynamics", Nuclear Science and Engineering, 126, 282-292 (1997). 
[20] V. V. KULIK, J. C. LEE, G. ALIBERTI, "Evaluation of different reactivity definitions for subcritical systems", Transaction of the American Nuclear Society, 89, 689-640 (2003).

[21] GANDINI, A. HGPT Based Sensitivity Time-Dependent Method for the Analysis of Subcritical Systems. Annals of Nuclear Energy, v. 28, pp. 1193-1217, 2001.

[22] A. GANDINI, "ADS subcriticality evaluation based on the generalized reactivity concept", Annals of Nuclear Energy, 31, 813-821, (2004).

[23] S. PELLONI, P. CODDINGTON, "On using alternative perturbation theory methodologies in the evaluation of reactivity effects in ADS cores", Annals of Nuclear Energy, 33, 1360-1367, (2006).

[24] HENRY, A. F. The application of reactor kinetics to the analysis of experiments. Nuclear Science and Engineering, v.3, pp. 52, 1958.

[25] G. R. KEEPIN. Physics of Nuclear Kinetics. Addison-Wesley, 1965.

[26] LEWINS, J. The Time-dependent Importance on Neutrons and Precursors. Nuclear Science and Engineering, v.7, pp. 286-274, 1960.

[27] FODERARO, A.; GARABEDIAN, H. L. A New Method for the Solution of Group Diffusion Equations. Nuclear Science and Engineering, v.8, pp. 44-52, 1960.

[28] LEWINS, J. The Reduction of the Time-Dependent Equations for Nuclear Reactors to a set of Ordinary Differential Equations. Nuclear Science and Engineering, v.9, pp. 399-407, 1961.

[29] BECKER, M. A Generalized Formulation of the Point Nuclear Reactor Kinetics Equations. Nuclear Science and Engineering, v. 31, pp.458-464, 1968.

[30] OHTANI, N. et al., Solutions do Diffusion Equations by Fourier Expansions. Nuclear Science and Engineering, v. 1, pp. 547-553, 1974.

[31] CORNO, S. E.; RAVETTO, P.; SUMINI, M. Nuclear Reactor Kinetics with a Continues Slowing-Down Model. Annals of Nuclear Energy, v. 11, n. 11, pp. 547-557, 1984.

[32] LEE, C. E.; ROTTLER, S. Analytic Solutions of the Multigroup Space-Time Reactor Kinetics Equations-I. Annals of Nuclear Energy, v. 13, n. 5, pp. 245-268, 1986. 
[33] DUlla, S.; RAVETTO, PP.; ROSTAGnO, M. M. On Some Features of QuasiStatic Schemes in Reactor Kinetics. The Physics of Fuel Cycle and Advanced Nuclear Systems: Global Developments, PHYSOR’04, Chicago, April 25-29. LaGrange Park, IL: American Nuclear Society, 2004. CD-ROM.

[34] DULlA, S.; RAVETTO, P.; ROSTAGNO, M. M. Some Features of Spatial Neutron Kinetics for Multiplying Systems. Annals of Nuclear Energy, v. 149, pp. 34-89, 2005.

[35] DUlla, S; GANAPOL, B. D.; RAVETTO, P. Space Asymptotic Method for the Study of Neutron Propagation. Annals of Nuclear Energy, v. 33, pp. 932-940, 2006

[36] DULLA, S. et al. Analytical Benchmarks for the Kinetics of Accelerator-Driven Systems. Joint International Topical Meeting on Mathematics \& Computation and Supercomputing in Nuclear Applications (M\&C + SNA 2007), Monterey, California, April 15-19, 2007, American Nuclear Society, LaGrange Park, IL, 2007. CD-ROM.

[37] OLIVEIRA, F. L. de. Solução Analítica da Cinética Espacial do Modelo de Difusão para Sistemas Homogêneos Subcríticos acionados por Fonte Externa. 2008. Dissertação (Mestrado)-Instituto de Pesquisas Energéticas e Nucleares, São Paulo.

[38] M. SALVATORES et al., Experimental Investigation Multiplying subcritical media in presence of an external source operating in pulsed or continuous mode: the MUSE-3 Experiment.

[39] CARL-MAGNUS PERSSON et al., Analysis of Reactivity determination methods in the subcritical experiment Yalina. Nuclear Instruments and Methods in Physics Research. A 554 (2005) 374-383.

[40] J. DUDERSTADT; J. L. HAMILTON. Nuclear Reactor Analysis. [S. 1.]: John Willey, 1972.

[41] A. F. HENRY, Nuclear Reactor Analysis, MIT Press, Cambridge, MA (1975).

[42] G. I. BELL \& S. CLASSTONE, Nuclear Reactor Theory, Van Nostrand Reinhold, New York, 1979

[43] A. GANDINI \& M.SALVATORES, The Physics of Sub critical Multiplying System, Journal of Nuclear Science and Technology, 39(6), 673-686, 2002.

[44] JOHN R. LAMARSH. Introduction to Nuclear Reactor Theory. Addison-Wesley, 1972.

[45] ADMIR DOS SANTOS, Comunicação pessoal. 
[46] G.D. SPRING, In-Pile measurement of the Decay Constant and Relative Abundances of Delayed Neutrons, Nucl. Sci. Eng. 114, 342351 (1993).

[47] A. DOS SANTOS et al., The application of the multiple transient for the experimental determination of the relative abundances and decay constant of delayed neutrons of the IPEN/MB-01 reactor, Annals of Nuclear Energy 33, 917-923 (2006).

[48] R. DINIZ et al., Experimental determination of the decay constants and abundances of delayed neutrons by means of reactor noise analysis. Nucl. Sci. Eng. 133, 314-326 (2006).

[49] R. KURAMOTO, Desenvolvimento de uma Metodologia Baseada no Modelo de Duas-Regiões e em Técnicas de Análise de Ruío Microscópico para a Medida Absoluta dos Parâmetros Cinéticos $\beta_{\text {eff, }} \Lambda$ e $\beta_{\text {eff }} / \Lambda$ do Reator IPEN/MB-01. Tese de doutorado IPEN. (2007).

[50] CARPENTER, S. G., Measurements of the Effective Delayed-Neutron Fraction in two Fast Critical Experiments. Nucl. Sci. Eng. 49, 235. (1972).

[51] FISHER E. A., Integral Measurements of the Effective Delayed Neutron Fractions in the Fast Critical Assembly SNEAK. Nucl. Sci. Eng. 62, 105. (1977).

[52] AVRAMOV, A. M., et. al. (1990) The Measurements of the Effective DelayedNeutron Fraction in the Fast Critical Assembly BFS with Uranium-Plutonium Metal Fuel, Proc. Int. Conf. on the Physics of Reactors. Marseille, France.

[53] BERTRAND, P., Bernice-Interlaboratory Comparison of $\beta_{\text {eff }}$ Measurement Techniques at MASURCA. Proc. Int. Conf. on the Physics of Reactors. Mito, Japan, 190199. (1996).

[54] CHAUSSONET, P. et al. International $\beta_{\text {eff }}$ benchmark experiment in fca cea results. Prog. Nucl. Energy, v. 35, pp. 157-162, 1999.

[55] V. A. DULIN, G. M. MIKHAILOV, Rossi- $\alpha$ Measurement of the Effective Delayed Neutron Fraction. Atomic Energy, Vol. 78, No. 3, 1995.

[56] R. E. UHRIG. Random Noise Techiques in Nuclear Reactor Systems. [S.1.]: The Ronald Press Company, 1970.

[57] M.M.R.WILLIAMS. Random Processes in Nuclear Reactor. [S.1.]: Pergamon,1974.

[58] J. A.THIE. Reactor Noise. [S.1.]: Rowman and Littlefield, Inc., 1963.

[59] R. P. FEYNMANN; F. HOFFMAN; R. SERBER. J.Nucl.Energy, v. 3, pp. 64, 1956.

[60] W. MATTHES. Nucleonik, v. 4, pp. 213, 1962.

[61] H.BORGWALDT; D. STEGEMANN. Nucleonik, v. 7, pp. 313, 1965 
[63] T. IIJIMA. Nucleonik, v. 10, pp. 93, 1967.

[64] SPRIGGS, G. D.; SAKURAI, T.; OKAJIMA, S. Rossi- $\alpha$ and $\beta_{\text {eff }}$ Measurements in a Fast Critical Assembly. Prog. Nucl. Energy, v. 35, n. 2, pp. 169-181, 1999.

[65] SRPIGGS, G. D., Two Rossi- $\alpha$ Techniques for Measuring the Effective Delayed Neutron Fraction. Nucl. Sci. Eng., 113, 161 (1993).

[66] SRPIGGS, G. D.; BUSCH R. D. B.; SAKURAI T.; OKAJIMA S., The Equivalent Fundamental-Mode Source. Annals of Nuclear Energy, 26, 237 (1999).

[67] MISAWA, T.; SHIROYA, S.; KANDA, K. Measurement of prompt neutron decay constant and large subcriticality by the Feynman- $\alpha$ method. Nucl. Sci. and Eng., v. 104, pp. 53, 1962.

[68] KITAMURA, Y. et al. General formulae for the Feynman- $\alpha$ method with the bunching technique. Ann. Nucl. Energy, v. 27, pp. 1199, 2000.

[69] YAMANE, Y.; TAKEMOTO, Y.; IMAI, T. Effective delayed neutron fraction measurements in fca-xix cores by using modified Bennett method. Prog. Nucl. Energy, v. 35, n. 2, pp. 183-194, 1999.

[70] BENNETT, E. F., An Experimental Method for Reactor-Noise Measurements of Effective Beta, ANL-81-72, 1981.

[71] YAMANE, Y., IIJIMA, T., SHIROYA, S. J. At. Energy Soc. Japan, 37(6), 513. 1995.

[72] OTSUKA, M., IIJIMA,T., Nukleonik, 7(8), 488. 1965.

[73] B. E. SIMMONS; J. S. KING, Nucl. Sci. Eng. 3 (1958) 595.

[74] N. G. SJÖSTRAND, Ark, Fys. 11 (1956) 13. 


\section{APÊNDICE}

\section{A.1 TABELAS DOS RESULTADOS}

Os parâmetros cinéticos foram calculados para vários valores de $K$ e os valores encontram-se nas duas tabelas abaixo, onde $K_{c}$ e $K_{s}$ denotam os fatores de multiplicação do sistema crítico e subcrítico, respectivamente.

Tabela A.1.1 Os parâmetros cinéticos do sistema rápido

\begin{tabular}{|c|c|c|c|c|c|c|c|c|}
\hline$K_{c}$ & $\beta$ & $\Lambda$ & $\rho$ & $\boldsymbol{K}_{s}$ & $\boldsymbol{\beta}_{s}$ & $\Lambda_{s}$ & $\rho_{0, s}$ & $\rho_{s}$ \\
\hline 1 & 0.0045 & $2.721 \mathrm{E}-06$ & 0 & 0.9894138 & 0.004458776 & $2.692 \mathrm{E}-06$ & -0.010579 & -0.005962 \\
\hline 0.997627 & 0.0045 & $2.721 \mathrm{E}-06$ & -0.002379 & 0.9870907 & 0.004448307 & $2.686 \mathrm{E}-06$ & -0.012898 & -0.008269 \\
\hline 0.9952653 & 0.0045 & $2.721 \mathrm{E}-06$ & -0.004757 & 0.9847785 & 0.004437889 & $2.68 \mathrm{E}-06$ & -0.015206 & -0.010565 \\
\hline 0.9929147 & 0.0045 & $2.721 \mathrm{E}-06$ & -0.007136 & 0.9824772 & 0.004427524 & $2.673 \mathrm{E}-06$ & -0.017503 & -0.012849 \\
\hline 0.9894096 & 0.0045 & $2.721 \mathrm{E}-06$ & -0.010704 & 0.9790452 & 0.004412073 & $2.664 \mathrm{E}-06$ & -0.020926 & -0.016254 \\
\hline 0.9859291 & 0.0045 & $2.721 \mathrm{E}-06$ & -0.014272 & 0.9756371 & 0.004396735 & $2.655 \mathrm{E}-06$ & -0.024324 & -0.019634 \\
\hline 0.9836223 & 0.0045 & $2.721 \mathrm{E}-06$ & -0.01665 & 0.9733782 & 0.004386574 & $2.649 \mathrm{E}-06$ & -0.026576 & -0.021874 \\
\hline 0.9813264 & 0.0045 & $2.721 \mathrm{E}-06$ & -0.019029 & 0.9711298 & 0.004376462 & $2.643 \mathrm{E}-06$ & -0.028816 & -0.024103 \\
\hline 0.9790411 & 0.0045 & $2.721 \mathrm{E}-06$ & -0.021408 & 0.9688917 & 0.0043664 & $2.637 \mathrm{E}-06$ & -0.031045 & -0.02632 \\
\hline 0.9767664 & 0.0045 & $2.721 \mathrm{E}-06$ & -0.023786 & 0.9666639 & 0.004356386 & $2.631 \mathrm{E}-06$ & -0.033264 & -0.028527 \\
\hline 0.9745023 & 0.0045 & $2.721 \mathrm{E}-06$ & -0.026165 & 0.9644463 & 0.004346422 & $2.625 \mathrm{E}-06$ & -0.035472 & -0.030723 \\
\hline 0.9722487 & 0.0045 & $2.721 \mathrm{E}-06$ & -0.028543 & 0.9622389 & 0.004336506 & $2.619 \mathrm{E}-06$ & -0.037669 & -0.032908 \\
\hline 0.9700054 & 0.0045 & $2.721 \mathrm{E}-06$ & -0.030922 & 0.9600415 & 0.004326639 & $2.613 \mathrm{E}-06$ & -0.039855 & -0.035083 \\
\hline 0.9677725 & 0.0045 & $2.721 \mathrm{E}-06$ & -0.033301 & 0.9578542 & 0.004316819 & $2.607 \mathrm{E}-06$ & -0.042031 & -0.037247 \\
\hline 0.9655498 & 0.0045 & $2.721 \mathrm{E}-06$ & -0.035679 & 0.9556768 & 0.004307046 & $2.601 \mathrm{E}-06$ & -0.044196 & -0.039401 \\
\hline 0.9633374 & 0.0045 & $2.721 \mathrm{E}-06$ & -0.038058 & 0.9535093 & 0.00429732 & $2.595 \mathrm{E}-06$ & -0.046351 & -0.041545 \\
\hline 0.9589427 & 0.0045 & $2.721 \mathrm{E}-06$ & -0.042815 & 0.9492037 & 0.004278009 & $2.583 \mathrm{E}-06$ & -0.05063 & -0.045801 \\
\hline 0.9545879 & 0.0045 & $2.721 \mathrm{E}-06$ & -0.047572 & 0.9449367 & 0.004258881 & $2.572 \mathrm{E}-06$ & -0.054869 & -0.050017 \\
\hline 0.9438721 & 0.0045 & $2.721 \mathrm{E}-06$ & -0.059466 & 0.9344353 & 0.004211849 & $2.543 \mathrm{E}-06$ & -0.06529 & -0.060383 \\
\hline 0.9333942 & 0.0045 & $2.721 \mathrm{E}-06$ & -0.071359 & 0.9241647 & 0.00416591 & $2.516 \mathrm{E}-06$ & -0.07547 & -0.070508 \\
\hline 0.9231464 & 0.0045 & $2.721 \mathrm{E}-06$ & -0.083252 & 0.9141175 & 0.004121024 & $2.489 \mathrm{E}-06$ & -0.085417 & -0.080401 \\
\hline 0.9131212 & 0.0045 & $2.721 \mathrm{E}-06$ & -0.095145 & 0.9042864 & 0.004077156 & $2.462 \mathrm{E}-06$ & -0.095139 & -0.090071 \\
\hline 0.9033114 & 0.0045 & $2.721 \mathrm{E}-06$ & -0.107038 & 0.8946645 & 0.004034269 & $2.436 \mathrm{E}-06$ & -0.104644 & -0.099524 \\
\hline 0.8937101 & 0.0045 & $2.721 \mathrm{E}-06$ & -0.118931 & 0.8852452 & 0.00399233 & $2.411 \mathrm{E}-06$ & -0.113939 & -0.108768 \\
\hline 0.8843108 & 0.0045 & $2.721 \mathrm{E}-06$ & -0.130824 & 0.8760221 & 0.003951308 & $2.386 \mathrm{E}-06$ & -0.12303 & -0.11781 \\
\hline
\end{tabular}


\begin{tabular}{|l|l|l|l|l|l|l|l|l|l|}
0.8751071 & 0.0045 & $2.721 \mathrm{E}-06$ & -0.142717 & & 0.8669893 & 0.003911172 & $2.362 \mathrm{E}-06$ & -0.131927 & -0.126658 \\
\hline
\end{tabular}

Tabela A.1.2 Os parâmetros cinéticos do sistema térmico.

\begin{tabular}{|c|c|c|c|c|c|c|c|c|}
\hline$K_{c}$ & $\beta$ & $\Lambda$ & $\rho$ & $\boldsymbol{K}_{s}$ & $\boldsymbol{\beta}_{s}$ & $\Lambda_{s}$ & $\rho_{0, s}$ & $\rho_{s}$ \\
\hline 1 & 0.0065 & $2.44 \mathrm{E}-05$ & 0 & 0.98 & 0.006413 & $2.41 \mathrm{E}-05$ & -0.01332 & -0.00035 \\
\hline 0.995139 & 0.0065 & $2.44 \mathrm{E}-05$ & -0.00489 & 0.975242 & 0.006393 & $2.4 \mathrm{E}-05$ & -0.01652 & -0.00353 \\
\hline 0.990324 & 0.0065 & $2.44 \mathrm{E}-05$ & -0.00977 & 0.97053 & 0.006372 & $2.4 \mathrm{E}-05$ & -0.01971 & -0.0067 \\
\hline 0.985556 & 0.0065 & $2.44 \mathrm{E}-05$ & -0.01466 & 0.965864 & 0.006351 & 2.39E-05 & -0.02288 & -0.00984 \\
\hline 0.980834 & 0.0065 & $2.44 \mathrm{E}-05$ & -0.01954 & 0.961242 & 0.006331 & $2.38 \mathrm{E}-05$ & -0.02603 & -0.01297 \\
\hline 0.976157 & 0.0065 & $2.44 \mathrm{E}-05$ & -0.02443 & 0.956664 & 0.00631 & $2.37 \mathrm{E}-05$ & -0.02916 & -0.01609 \\
\hline 0.971524 & 0.0065 & $2.44 \mathrm{E}-05$ & -0.02931 & 0.95213 & 0.00629 & $2.37 \mathrm{E}-05$ & -0.03228 & -0.01918 \\
\hline 0.966935 & 0.0065 & $2.44 \mathrm{E}-05$ & -0.0342 & 0.947638 & 0.00627 & $2.36 \mathrm{E}-05$ & -0.03538 & -0.02226 \\
\hline 0.962389 & 0.0065 & $2.44 \mathrm{E}-05$ & -0.03908 & 0.943189 & 0.00625 & $2.35 \mathrm{E}-05$ & -0.03846 & -0.02532 \\
\hline 0.957886 & 0.0065 & $2.44 \mathrm{E}-05$ & -0.04397 & 0.938781 & 0.00623 & 2.34E-05 & -0.04152 & -0.02836 \\
\hline 0.953424 & 0.0065 & $2.44 \mathrm{E}-05$ & -0.04885 & 0.934414 & 0.00621 & 2.34E-05 & -0.04457 & -0.03139 \\
\hline 0.949004 & 0.0065 & $2.44 \mathrm{E}-05$ & -0.05374 & 0.930088 & 0.006191 & 2.33E-05 & -0.0476 & -0.0344 \\
\hline 0.944625 & 0.0065 & $2.44 \mathrm{E}-05$ & -0.05862 & 0.925801 & 0.006171 & 2.32E-05 & -0.05062 & -0.03739 \\
\hline 0.940286 & 0.0065 & $2.44 \mathrm{E}-05$ & -0.06351 & 0.921554 & 0.006152 & 2.31E-05 & -0.05361 & -0.04037 \\
\hline 0.935987 & 0.0065 & 2.44E-05 & -0.06839 & 0.917345 & 0.006132 & $2.31 \mathrm{E}-05$ & -0.05659 & -0.04333 \\
\hline 0.931727 & 0.0065 & $2.44 \mathrm{E}-05$ & -0.07328 & 0.913175 & 0.006113 & $2.3 \mathrm{E}-05$ & -0.05956 & -0.04627 \\
\hline 0.927505 & 0.0065 & $2.44 \mathrm{E}-05$ & -0.07816 & 0.909043 & 0.006094 & $2.29 \mathrm{E}-05$ & -0.0625 & -0.0492 \\
\hline
\end{tabular}




\section{A.2 OPERADORES ADJUNTOS}

Para defenir o que são operadores adjuntos, é necessário definir, primeiro, o produto interno. O produto interno de duas funções $f(\boldsymbol{r})$ e $g(\boldsymbol{r})$ é definido da seguinte maneira:

$$
\langle f, g\rangle \equiv \int_{V} f^{+}(\boldsymbol{r}) g(\boldsymbol{r}) \mathrm{d} \boldsymbol{r}
$$

onde $f^{+}(\boldsymbol{r})$ denota o complexo conjugado da função $f(\boldsymbol{r})$, e $V$ é o volume do sistema. Se f e g são funções que dependem do espaço, energia e direção, i. e., como no caso discutido na Seção 2.2, o produto interno é definido como o integral em todo o espaço da fase, como é mostrado na Expressão (2.12)

O operdor adjunto ao operador $\mathrm{M}$ é o complexo conjudado do $\mathrm{M}$, e é denotado como $\mathrm{M}^{+}$, e satisfaz a seguinte igualdade:

$$
\left\langle M^{+} f, g\right\rangle=\langle f, M g\rangle
$$

ou seja,

$$
\int(M f)^{+} g d \boldsymbol{r}=\int f^{+} M g d \boldsymbol{r}
$$

para quaiquer funções $f$ e $g$, que satisfazem a mesma condição de contorno, por exemplo, $f\left(\boldsymbol{r}_{s}\right)=g\left(\boldsymbol{r}_{s}\right)=0$.

Se o operador M é uma matriz quadrada de ordem $n$, i. e.:

$$
\mathbf{M}=\left[\begin{array}{ccc}
M 11 & \cdots & M 1 n \\
\vdots & \ddots & \vdots \\
M n 1 & \cdots & M n n
\end{array}\right]
$$

o complexo conjugado, $\mathrm{M}^{*}$, é obtido construindo uma matriz, cujos elementos são complexos conjudados aos correspondentes de M, ou seja: 


$$
\mathrm{M}^{*}=\left[\begin{array}{ccc}
M 11 * & \cdots & M 1 n * \\
\vdots & \ddots & \vdots \\
M n 1 * & \cdots & M n n *
\end{array}\right]
$$

e o adjunto do operador $\mathrm{M}$, i. e., $\mathrm{M}^{+}$, é dado como a transposta dessa matriz $\mathrm{M}^{*}$ :

$$
\mathbf{M}^{+}=\left[\begin{array}{ccc}
M 11 * & \cdots & M 1 * \\
\vdots & \ddots & \vdots \\
M 1 n * & \cdots & M n n *
\end{array}\right]
$$

Diz-se que um operador é auto-adjunto ou hermitiano, quando o mesmo é idêntico ao seu adjunto, isto é: $\mathrm{M}=\mathrm{M}^{+}$. Uma matriz diagonal, por exemplo, cujos elemento são todos reais, é hermitiano. Note que, uma matriz real nem sempre é hermitiana. E, é claro, uma grandeza real, e. g, $v \Sigma_{f}$, é, também, um operador auto-adjunto. 


\section{A.3 TEORIA DA PERTURBAÇÃo}

Se um sistema sofrer uma variação, tanto na geometria como na composição, e se essa variação for suficientemente pequena, o efeito desta pequna variação ou "perturbação" poderá ser expresso em termos de fluxos do estado original usando a teoria de perturbação.

Dada a equação da criticalidade:

$$
L \Phi=\frac{1}{k} M \Phi
$$

suponha que a seção de choque macroscópica de absorção fosse perturbada. A nova seção de choque de absorção terá um valor:

$$
\Sigma_{a}=\Sigma_{a}+\delta \Sigma_{a}
$$

onde $\delta \Sigma_{a}$ denota a perturbação em $\Sigma_{a}$, e assume-se que $\delta \Sigma_{a} \ll<\Sigma_{a}$. Note que esta perturbação aparece como uma perturbação $\delta L$ no operador $L$. Portanto:

$$
M{ }^{\prime}=M+\delta M
$$

O sitema perturbado é, então, caracterizado por uma nova equação:

$$
L^{\prime} \Phi=\frac{1}{k^{\prime}} M \Phi^{`}
$$

Aplicando (A.2.3) em (A.2.1), e tomando o produto interno da (A.2.1) com $\Phi^{+}$, a adjunta de $\Phi$, e usando a definição dos operadores adjuntos (A.1.2), obtém-se a seguinte igualdade: 


$$
\left(\frac{1}{k^{`}}-\frac{1}{k}\right)=\frac{\left\langle\Phi^{+}, \delta L \Phi^{\dagger}\right\rangle}{\left\langle\Phi^{+}, M \Phi^{\dagger}\right\rangle}
$$

Agora, se é pequeno, $\delta \Sigma_{a}$ é pequeno, $\delta \Phi$ também. Portanto:

$$
\left(\frac{1}{k^{`}}-\frac{1}{k}\right)=\frac{\left\langle\Phi^{+}, \delta L \Phi\right\rangle}{\left\langle\Phi^{+}, M \Phi\right\rangle}+\frac{\left\langle\Phi^{+}, \delta L \delta \Phi\right\rangle}{\left\langle\Phi^{+}, M \Phi\right\rangle}-\frac{\left\langle\Phi^{+}, \delta L \Phi\right\rangle\left\langle\Phi^{+}, M \delta \Phi\right\rangle}{\left\langle\Phi^{+}, M \Phi\right\rangle^{2}}+\ldots .
$$

Usando a teoria de perturbação de $1^{\mathrm{a}}$ ordem, ou seja, desprezando todos $\delta$ de ordem igual ou superior a $\delta^{2}$, chega-se a seguinte equação:

$$
\left(\frac{1}{k^{`}}-\frac{1}{k}\right)=\frac{\left\langle\Phi^{+}, \delta L \Phi\right\rangle}{\left\langle\Phi^{+}, M \Phi\right\rangle}
$$

Desta forma, foi possível calcular $k$, que é a característica no sistema do estado perturbado, em termos de constantes e fluxos do estado não perturbado, sem precisar repetir o cálculo do problema de criticalidade (A.2.1), usando a teoria da perturbação. Note que este tipo de procedimento não é restrito ao cálculo de $k$.

Introduzindo o parâmetro defindo como:

$$
\rho \equiv \frac{(k-1)}{k},
$$

a (A.2.7) pode ser escrita como:

$$
\Delta \rho \equiv \rho^{`}-\rho \cong \frac{\left\langle\Phi^{+}, \delta L \Phi\right\rangle}{\left\langle\Phi^{+}, M \Phi\right\rangle}
$$

